CLNS 96/1442; hep-th/9701016

\title{
The Scattering Theory of Oscillator Defects in an Optical Fiber
}

\author{
Robert Konik and André LeClair \\ Newman Laboratory \\ Cornell University \\ Ithaca, NY 14853
}

\begin{abstract}
We examine harmonic oscillator defects coupled to a photon field in the environs of an optical fiber. Using techniques borrowed or extended from the theory of two dimensional quantum fields with boundaries and defects, we are able to compute exactly a number of interesting quantities. We calculate the scattering S-matrices (i.e. the reflection and transmission amplitudes) of the photons off a single defect. We determine using techniques derived from thermodynamic Bethe ansatz (TBA) the thermodynamic potentials of the interacting photon-defect system. And we compute several correlators of physical interest. We find the photon occupancy at finite temperature, the spontaneous emission spectrum from the decay of an excited state, and the correlation functions of the defect degrees of freedom. In an extension of the single defect theory, we find the photonic band structure that arises from a periodic array of harmonic oscillators. In another extension, we examine a continuous array of defects and exactly derive its dispersion relation. With some differences, the spectrum is similar to that found for EM wave propagation in covalent crystals. We then add to this continuum theory isolated defects, so as to obtain a more realistic model of defects embedded in a frequency dependent dielectric medium. We do this both with a single isolated defect and with an array of isolated defects, and so compute how the S-matrices and the band structure change in a dynamic medium.
\end{abstract}

$12 / 96$ 


\section{Introduction}

In this paper we examine photons interacting with harmonic oscillator impurities in an optical fiber. Because this problem is inherently two dimensional, we are able to attack it by applying or extending various techniques from two dimensional defect and boundary field theory ([1] [2] [3]). This permits us to compute exactly quantities previously inaccessible. Here, for example, using methods akin to thermodynamic Bethe ansatz (TBA), we compute thermodynamic potentials. And through extending the notion of boundary states, we exactly compute correlators.

Normally the impurity atoms in optical problems are modeled as two-level systems. Although this makes the problem non-linear, some success has been had in finding exact solutions in two dimensions. Under the rotating wave approximation, a continuum array of defects has been shown to be integrable (四). There, using algebraic Bethe ansatz, the spectrum and the S-matrices of the theory were found. In [5] it was shown that a single two-level atom can be mapped onto a Kondo impurity. With this mapping they were able to compute thermodynamic potentials exactly and to obtain form factor expansions of some correlators. But such successes are limited. Because of the theory's non-linearity, integrability is key to obtaining exact solutions. However integrability limits the type of defect configurations one can consider. Outside the rotating wave approximation, an array of two-level atoms is not known to be integrable. To then say something one must resort to perturbation theory (see [6]). And although a single two-level atom can be treated, two or some larger number cannot. Nor does integrability guarantee that all interesting physical quantities can be accessed. Photon correlators for two-level systems are still only known approximately. And the computation of correlators involving the impurity degrees of freedom remains an open problem. To sidestep these difficulties we choose to model the atoms as harmonic oscillators. In doing this we cease to be limited by particular configurations of atoms under certain approximations and we gain access to a wider variety of quantities, including both exact photon and impurity correlators.

The replacement of two-level atoms by harmonic oscillators is not necessarily a bad one. Providing the intensity of the photon field in the fiber is low, only the oscillator's ground and first excited state are likely to be occupied. Under these conditions, the oscillator mimics a two-level system. We will see later, for example, how the spontaneous emission spectrum mimics that of a two-level atom. However, we cannot hope to model such phenomena as strong field resonance fluorescence. 
We exploit our ability to study arbitrary configurations of defects by examining both periodic and continuum arrays of defects. With a periodic array of defects, a band structure of photons naturally appears. This band structure is equipped with gaps of two types: one arising from being on resonance and a more typical one of gaps appearing at the edges of the Brillouin zones.

In the case of the continuum array of defects, we derive a dispersion relation for the photons propagating among the defects. We find that the dressed photons are described by the typical polariton dispersion relation. Again exploiting our configurational flexibility, we examine theories where isolated defects are added to the continuum array. We look at two examples: an isolated defect and a periodic array of defects. With the isolated defect we compute the scattering matrices of the polaritons off the defect. In the case of the periodic array, we calculate the band structure obeyed by the polaritons.

The ability to consider defects living in a dynamic medium lays the ground for the consideration of photon localization. By choosing the energy of an isolated defect to lie within the gap between the two polariton branches, there is some chance to find a photon in a bound state. We will consider this possibility in an upcoming work.

The paper is organized as follows. In the first section an overview of the model as well as its reduction to two dimensions is given. In the next section the scattering matrices are derived, followed by a section giving the TBA analysis of the thermodynamic potentials. In the next section correlators are computed. There we compute both time ordered and non-time ordered correlators. The non-time ordered correlators, important as they often represent what can be physically measured, are accessed via analytic continuations of temperature correlators. In the next section we consider multiple defects and in particular periodic arrays of defects. In the final two sections, we consider a continuum array of defects, first alone, then together with embedded impurities.

\section{Overview of Model}

In this section we describe the coupling between a single harmonic oscillator defect (placed at the origin) and a photon field in the environs of an optical fiber. The oscillations of the defect have two possible physical sources. The first, going under the name "atomic polarizability", arises from the distortion of charge within an atom. One can crudely model this by representing the atom as a charged shell tied to an immobile nucleus through a spring of strength $K=m \omega_{0}^{2}$ ( $\mathrm{m}$ is the mass of the shell and equal to $Z m_{e}$ where $Z$ is 
the atomic number and $m_{e}$ is the mass of an electron). The shell then executes simple harmonic motion around the fixed nucleus. Typically the energy scale of these oscillations is $\hbar \omega_{0} \sim 10 \mathrm{eV}$.

The second source comes with the name, "displacement polarizability". Here the defect is considered as a pair of oppositely charged ions that oscillate about their centre of mass. Because these oscillations are phonon-like (i.e. the heavier ions are oscillating and not the electronic shell), their energy scale $\hbar \omega_{0}$ is typically $10^{-1} \sim 10^{-2} \mathrm{eV}$. In this section we will consider the defect to be of this latter type. At energy scales of the former, we would also need to consider the atomic polarizability of the fiber medium in which the photons propagate and in which the defects are embedded. Instead we are able to treat the medium of propagation as vacuum (or equally easily, a medium of static dielectric constant, $\left.\epsilon_{0}\right)$. We will delay the inclusion of the dynamic properties of the medium to Section 7 .

The oscillator, minimally coupled to the photon field in the radiation gauge, has the Hamiltonian

$$
H=\frac{\mathrm{p}^{2}}{2 m}+\frac{m \omega_{0}^{2}}{2} \mathrm{q}^{2}-\frac{\mathrm{pe}}{2 \mathrm{~m}} \mathbf{A}(\mathrm{x}=0)+\frac{\mathrm{e}^{2}}{2 \mathrm{mc}^{2}} \mathbf{A}^{2}(\mathrm{x}=0) .
$$

By now introducing, in the standard way, the variables

$$
\begin{aligned}
S^{ \pm} & = \pm i\left(\frac{m \omega_{0}}{2 \hbar}\right)^{1 / 2} \mathrm{q}+\frac{1}{\left(2 m \hbar \omega_{0}\right)^{1 / 2}} \mathrm{p} \\
1 & =\left[S^{-}, S^{+}\right]
\end{aligned}
$$

this Hamiltonian can be recast as

$$
H=\hbar \omega_{0}\left(S^{+} S^{-}+1 / 2\right)-\frac{e}{c}\left(\frac{\hbar \omega_{0}}{2 m}\right)^{1 / 2}\left(S^{+}+S^{-}\right) \mathbf{A}(x=0)+\frac{e^{2}}{2 m c^{2}} \mathbf{A}^{2}(x=0) .
$$

The interaction terms are then of two sorts: one coupling the photon field to the oscillator and one giving the photon a "mass" at $x=0$. Throughout our analysis we will keep this latter term. The $\mathbf{A}^{2}$ term contributes equally to the quantities which we will be interested in calculating. Its omission, on the other hand, thereby violating gauge invariance, leads to unphysical results. Fortunately, its inclusion does not overly complicate the analysis.

As we ultimately want to derive equations of motion, we want to write the above as an action. Because $S^{+}$and $S^{-}$can be thought of as conjugate variables, i.e. $\left[i \hbar S^{-}, S^{+}\right]=i \hbar$, 
this can be easily done. The action is

$$
\begin{aligned}
S_{s h o}= & \int d t\left(-i \hbar S^{-} \partial_{t} S^{+}-H\right) \\
= & \int d t\left(-i \hbar S^{-} \partial_{t} S^{+}-\hbar \omega_{0} S^{+} S^{-}\right. \\
& \left.+\frac{e}{c}\left(\frac{\hbar \omega_{0}}{2 m}\right)^{1 / 2}\left(S^{+}+S^{-}\right) \mathbf{A}(x=0)-\frac{e^{2}}{2 m c^{2}} \mathbf{A}^{2}(x=0)\right) .
\end{aligned}
$$

In writing this we have dropped the zero point energy of the oscillator.

We now focus our attention on the free dynamics of the photons. The action determining these dynamics is

$$
S_{A}=-\frac{1}{8 \pi} \int d^{4} x F^{\mu \nu} F_{\mu \nu}
$$

For a fiber, $S_{A}$ can be dimensionally reduced. We begin by setting the scalar potential $A_{0}$ to zero. We then take the fiber along the $\widehat{x}$-direction. A is necessarily in some transverse direction. Assuming only one direction of $\mathbf{A}$ is excited, say $\widehat{\mathbf{n}}$ where $\widehat{\mathbf{n}} \cdot \widehat{\mathbf{x}}=\mathbf{0}$, we can write $\mathbf{A}=A \widehat{\mathbf{n}}$. If $A$ is independent of the transverse directions, $\widehat{\mathbf{y}}$ and $\widehat{\mathbf{z}}, S_{A}$ reduces to

$$
S_{A}=\frac{D}{8 \pi} \int d t d x\left(c^{-2}\left(\partial_{t} A\right)^{2}-\left(\partial_{x} A\right)^{2}\right)
$$

where $\mathrm{D}$ is the cross-sectional area of the fiber arising from performing the integral $\int d y d z=$ $D$. We can rescale $A$ to turn $S_{A}$ into an action describing a 2-d free massless boson:

$$
A \rightarrow \frac{\phi}{D^{1 / 2}}
$$

so that

$$
S_{A}=\frac{1}{8 \pi} \int d t d x\left(c^{-2}\left(\partial_{t} \phi\right)^{2}-\left(\partial_{x} \phi\right)^{2}\right) .
$$

We list factors of $c$ and $\hbar$ so as to be able to easily provide estimates of various couplings.

The full action we consider is then

$$
\begin{array}{r}
S=S_{A}+S_{s h o}=\frac{1}{8 \pi} \int d t d x\left(c^{-2}\left(\partial_{t} \phi\right)^{2}-\left(\partial_{x} \phi\right)^{2}\right)+\int d t\left(-i \hbar S^{-} \partial_{t} S^{+}\right. \\
\left.-\hbar \omega_{0} S^{+} S^{-}+(\hbar c)^{1 / 2} \lambda\left(S^{+}+S^{-}\right) \phi-\frac{c}{\hbar} \Delta^{2} \phi^{2}\right) .
\end{array}
$$

where $\lambda$ and $\Delta^{2}$ are

$$
\begin{aligned}
\lambda & =\left(\frac{\omega_{0}}{2 m c D}\right)^{1 / 2} \frac{e}{c} \\
\Delta^{2} & =\frac{e^{2} \hbar}{2 m c^{3} D} .
\end{aligned}
$$


$\Delta^{2}$ has units of mass and $\lambda$ has units of inverse length. Because the bound, approximately by $10^{-4}$, on the fiber diameter, $D$, is large compared to atomic length scales, both $\lambda$ and $\Delta^{2}$ are small compared to the scale set by $\hbar \omega_{0} \sim 10^{-2}$ ev, the typical oscillator energy. We have

$$
\begin{aligned}
\frac{\lambda \hbar c}{\hbar \omega_{0}} & \sim 10^{-7}, \\
\frac{\Delta^{2} c^{2}}{\hbar \omega_{0}} & \sim 10^{-14} .
\end{aligned}
$$

We thus see that the mass perturbation $\Delta^{2}$ is on the same order as $\lambda^{2}$, as required by gauge invariance. When $\hbar=c=1$ the two scales are governed by

$$
\lambda^{2}=\Delta^{2} \omega_{0}
$$

Thus $\Delta^{2}$ cannot be ignored in favour of $\lambda$, the photon-oscillator coupling. Because of (2.12), we will express all derived quantities such as scattering matrices, band structure, correlators, etc., in terms of $\Delta^{2}$.

Though both couplings are small compared to $\hbar \omega_{0}$, the perturbations are still able to lead to interesting physics. It is possible to introduce other length scales into the problem by considering multiple defects, which in combination with the existing scales can lead to observable effects. We will consider such cases in Sections 6, 7, and 8. But for now we continue to develop the single defect formalism.

\section{Calculation of Defect S-Matrices}

Generally given a theory with particles $A(k)$, an integrable defect perturbation produces scattering via the defect S-matrices, $T(k)$ and $R(k)$, as follows:

$$
\mathbf{D} A_{+}(k)=T(k) A_{-}(k) \mathbf{D}+R(k) \mathbf{D} A_{+}(-k),
$$

where $\mathbf{D}$ is the defect operator. $\mathbf{D}$ is analogous to the boundary operator $\mathbf{B}$ discussed in [2]. $A_{ \pm}$represents particles who live on the right $(x>0) /$ left $(x<0)$ side of the defect. $T(k)$ is then just the amplitude that the particle, $A_{+}(k)$, makes it across the defect, and $R(k)$, the amplitude that it reflects back.

To derive $T$ and $R$ we reconsider the action in (2.9). Setting $\hbar=c=1$ and transforming to Euclidean time, $t \rightarrow-i t$, this becomes

$$
\begin{array}{rl}
S=\frac{1}{8 \pi} \int d & d x\left(\left(\partial_{t} \phi\right)^{2}+\left(\partial_{x} \phi\right)^{2}\right) \\
& +\int d t \omega_{0} S^{+} S^{-}-S^{-} \partial_{t} S^{+}-\lambda\left(S^{-}+S^{+}\right) \phi+\Delta^{2} \phi^{2} .
\end{array}
$$


By varying $S^{-}, S^{+}$, and $\phi$, we obtain the following equations of motion:

$$
\begin{aligned}
& 0=\lambda \delta(x)\left(S^{-}+S^{+}\right)+\frac{1}{4 \pi}\left(\partial_{t}^{2} \phi+\partial_{x}^{2} \phi\right)-2 \delta(x) \Delta^{2} \phi \\
& 0=\delta(x)\left(-\lambda \phi+\omega_{0} S^{ \pm} \mp \partial_{t} S^{ \pm}\right) .
\end{aligned}
$$

The second of these two has the steady-state solution

$$
S^{ \pm}=\lambda \int_{-\infty}^{\infty} d k e^{k t} \frac{\widetilde{\phi}(k)}{\omega_{0} \mp k}
$$

where $\widetilde{\phi}(k)$ is defined through

$$
\phi(x=0)=\int_{-\infty}^{\infty} d k e^{k t} \widetilde{\phi}(k)
$$

We can thus eliminate $S^{ \pm}$from the first equation of motion to obtain

$$
0=2 \omega_{0} \lambda^{2} \delta(x) \int_{-\infty}^{\infty} d k e^{k t} \frac{\widetilde{\phi}(k)}{\omega_{0}^{2}-k^{2}}+\frac{1}{4 \pi}\left(\partial_{t}^{2} \phi+\partial_{x}^{2} \phi\right)-2 \Delta^{2} \phi \delta(x) .
$$

To solve this equation we write $\phi$ as

$$
\phi(x)=\phi_{+}(x) \theta(x)+\phi_{-}(x) \theta(-x),
$$

where $\phi_{ \pm}$are free massless bosons with mode expansions

$$
\phi_{ \pm}(x)=\int_{-\infty}^{\infty} d k \frac{1}{(|k|)^{1 / 2}}\left(A_{ \pm}(k) e^{-t|k|+i x k}+A_{ \pm}^{\dagger}(k) e^{t|k|-i x k}\right) .
$$

We take $\phi(x=0)$ to be defined as $\phi(0)=1 / 2\left(\phi_{+}(0)+\phi_{-}(0)\right)$. So our equation of motion can be written as

$$
0=\omega_{0} \lambda^{2} \int_{-\infty}^{\infty} d k e^{k t} \frac{\widetilde{\phi}_{+}+\widetilde{\phi}_{-}}{\omega_{0}^{2}-k^{2}}+\frac{1}{4 \pi}\left(\partial_{x} \phi_{+}(0)-\partial_{x} \phi_{-}(0)\right)-\Delta^{2}\left(\phi_{+}(0)+\phi_{-}(0)\right) .
$$

Second order derivatives have vanished because $\left(\partial_{t}^{2}+\partial_{x}^{2}\right) \phi_{ \pm}=0$.

To derive $T(k)$ and $R(k)$, we divide the above equation into its '+' and '-' parts, say $f\left(A_{+}(k)\right)+g\left(A_{-}(k)\right)=0$, and so interpret it as vanishing when acting on the defect operator in the following manner:

$$
\mathbf{D} f\left(A_{+}(k)\right)+g\left(A_{-}(k)\right) \mathbf{D}=0 \text {. }
$$


Note carefully the orderings of $\mathbf{D}$ and the $A_{+}$'s and $A_{-}$'s. Substituting the mode expansions into this equation together with the continuity equation

$$
\mathbf{D} \phi_{+}(0)=\phi_{-}(0) \mathbf{D},
$$

fixes $T(k)$ and $R(k)$ to be

$$
\begin{aligned}
T(k) & =\frac{i\left(k^{2}-\omega_{0}^{2}\right)}{i\left(k^{2}-\omega_{0}^{2}\right)-4 \pi \Delta^{2} k}, \\
R(k) & =\frac{4 \pi \Delta^{2} k}{i\left(k^{2}-\omega_{0}^{2}\right)-4 \pi \Delta^{2} k} .
\end{aligned}
$$

As a check on the validity of this result, we see $T(k)$ and $R(k)$ satisfy the defect unitarity conditions:

$$
\begin{aligned}
& T(k) R(-k)+T(-k) R(k)=0 ; \\
& R(k) R(-k)+T(k) T(-k)=1 .
\end{aligned}
$$

These conditions are easily derived through applying (3.1) twice, i.e. allowing the particles to scatter through the defect twice.

The analytic structure of $T(k)$ and $R(k)$ is unsurprising. Both have poles at

$$
k_{p}= \pm \omega_{0}\left(1-\frac{4 \pi^{2} \Delta^{4}}{\omega_{0}^{2}}\right)^{1 / 2}-i 2 \pi \Delta^{2}
$$

The real part of $k_{p}$ describes the energy splitting between two oscillator levels. This splitting has been shifted slightly from $\omega_{0}$ in a process analogous to the Lamb shift. Minus the imaginary part of $k_{p}$ equals the inverse lifetime of an excited state. We then have

$$
\tau^{-1}=-\operatorname{Im} k_{p}=2 \pi \Delta^{2} .
$$

This lifetime is what is similar to what is found in perturbative treatments using the master equation (see for example [7]). This is unsurprising. If we were to subject the theory to a perturbative analysis we would find that the photon self-energy (i.e. the vacuum polarization) consists of a single diagram of lowest order and so $\tau^{-1}$ should have no contributions higher than $\Delta^{2}$. A similar situation is found in perturbative analyses of the simple defect theories in [1].

The interpretation of this pole differs from the standard one in integrable Euclidean QFT. Normally the imaginary part of $k_{p}$ would be interpreted as related to the energy of an excited oscillator state and the real part as the decay time of the excited state. But 
here the roles have been reversed, as if we still were in Minkowski space. This standard interpretation arises from assuming that the pole in the S-matrix arises from an effective propagator of the form $\left(p^{2}+m^{2}\right)^{-1}$. But here this is not the case. In Minkowski space, the pole appears as $\sim\left(k^{2}-\omega_{0}^{2}\right)^{-1}$. Both $k$ and $\omega_{0}$ are energies. In going to Euclidean space, we then have $k \rightarrow-i k$ and $\omega_{0} \rightarrow-i \omega_{0}$. Thus the pole structure retains its form in Euclidean space.

\section{Thermodynamics}

Free photons in a cavity at a temperature $T$ are governed by the well-known Planck distribution:

$$
\rho(k) d k=\frac{d k}{2 \pi} \frac{1}{e^{k / T}-1} .
$$

The mean energy per unit length is given by

$$
U(T)=\int_{-\infty}^{\infty} d k|k| \rho(k)=\frac{\pi}{6} T^{2} .
$$

The above expression for $U(T)$ is the one-dimensional analog of the Stephan-Boltzman law. $U(T)$ can also be expressed as

$$
U(T)=\frac{T^{2}}{L} \partial_{T} \log Z,
$$

where $L$ is the length of the cavity, and

$$
\log Z=-\frac{L}{2 \pi} \int_{-\infty}^{\infty} d k \log \left(1-e^{-|k| / T}\right)
$$

is the free energy of the non-interacting photons. We will now compute the modifications of the above blackbody theory due to the presence of the impurity. We place the impurity at the origin at $x=0$, let the cavity have length $L,-L / 2<x<L / 2$, and further impose periodic boundary conditions at the ends of the cavity.

For a single impurity, we can fold the system so that it is formulated as a theory on the half line with the impurity on the boundary at $x=0$. This kind of folding was used in the work [8] in their study of edge states in the $\nu=1 / 3$ quantum Hall system. Let $\phi=\phi_{L}+\phi_{R}$, where $\phi_{L}(x+t)$ and $\phi_{R}(x-t)$ are the left and right-moving components of the massless scalar field in the bulk. Define even and odd fields as follows:

$$
\begin{aligned}
\phi_{L}^{e}(x, t) & =\left(\phi_{L}(x, t)+\phi_{R}(-x, t)\right) / \sqrt{2} \\
\phi_{L}^{o}(x, t) & =\left(\phi_{L}(x, t)-\phi_{R}(-x, t)\right) / \sqrt{2} ; \\
\phi_{R}^{e}(x, t) & =\phi_{L}^{e}(-x, t) ; \quad \phi_{R}^{o}(x, t)=-\phi_{L}^{o}(-x, t) .
\end{aligned}
$$


Also define $\phi^{e, o}=\phi_{L}^{e, o}+\phi_{R}^{e, o}$. Then the action (2.9) can be rewritten as a theory on the half-line $x<0$ :

$$
\begin{aligned}
S=\frac{1}{8 \pi} \int_{-\infty}^{\infty} d t \int_{-\infty}^{0} d x\left(\left(\partial \phi^{e}\right)^{2}+\left(\partial \phi^{o}\right)^{2}\right) & \\
& +\int_{-\infty}^{\infty} d t\left(-i S^{-} \partial_{t} S^{+}-\omega_{0} S^{+} S^{-}+\frac{\lambda}{\sqrt{2}}\left(S^{+}+S^{-}\right) \phi^{e}-\frac{\Delta^{2}}{2}\left(\phi^{e}\right)^{2}\right) .
\end{aligned}
$$

Note that $\phi^{e}$ and $\phi^{o}$ decouple, and only $\phi^{e}$ couples to the impurity.

The interaction of the even photons with the impurity at the boundary are now summarized by a reflection matrix $\mathcal{R}_{e}$. The algebra (3.1) now only has a reflection piece:

$$
\mathbf{B} A(k)=\mathcal{R}_{e}(k) \mathbf{B} A(-k),
$$

where $\mathbf{B}$ is a boundary operator. A similar computation to the one in section 3 gives

$$
\mathcal{R}_{e}(k)=\frac{i\left(k^{2}-\omega_{0}^{2}\right)+4 \pi \Delta^{2} k}{i\left(k^{2}-\omega_{0}^{2}\right)-4 \pi \Delta^{2} k}
$$

A similar reflection matrix describes the odd photons. However as the odd photons do not couple to the defect, it is trivial, equaling unity.

Thermodynamic properties can all be expressed in terms of $\mathcal{R}_{e}$. The quantization condition on the momentum $k$ of an even photon on the half-line of length $L / 2$ is

$$
e^{i k L} \mathcal{R}_{e}(k)=1, \quad k>0
$$

We need only consider $k>0$ since it is implicit that the wave-function satisfying the above quantization condition contains both a right-moving and reflected left-moving particle. Taking the $k$-derivative of the log of (4.9) yields

$$
\widetilde{\rho_{e}}(k)=\frac{1}{2 \pi}-\frac{i}{2 \pi L} \partial_{k} \log \mathcal{R}_{e}(k),
$$

where $L \tilde{\rho_{e}}(k) d k$ is the number of allowed states between $k$ and $k+d k$. Introducing a density of occupied levels $\rho_{e}(k)$, one has for the partition function of the even field

$$
\log Z_{e}=-\frac{U_{e}}{T}+\mathcal{S}_{e}
$$

where $U_{e}=L \int_{0}^{\infty} d k k \rho_{e}(k)$ and $\mathcal{S}_{e}$ is the entropy:

$$
\mathcal{S}_{e}=L \int_{0}^{\infty} d k\left(\rho_{e}+\widetilde{\rho_{e}}\right) \log \left(\rho_{e}+\widetilde{\rho_{e}}\right)-\rho_{e} \log \rho_{e}-\tilde{\rho_{e}} \log \tilde{\rho_{e}} .
$$


Minimizing $\log Z_{e}$ with respect to $\rho_{e}$, one finds that the Planck distribution (4.1) for the even photons is modified to

$$
\rho_{e}(k) d k=\frac{1}{e^{k / T}-1}\left(\frac{1}{2 \pi}-\frac{i}{2 \pi L} \partial_{k} \log \mathcal{R}_{e}(k)\right) d k
$$

and the even free energy becomes

$$
\log Z_{e}=-L \int_{0}^{\infty} \tilde{\rho_{e}}(k) \log \left(1-e^{-k / T}\right)
$$

We now put this all together to find a total energy for the system.

The mean energy density separates into bulk and impurity contributions:

$$
U(T)=U_{\mathrm{bulk}}(T)+U_{\mathrm{imp}}(T) .
$$

$U_{\text {bulk }}$ is a sum of equal contributions from the even and odd sectors of the theory,

$$
U_{\mathrm{bulk}}=U_{\mathrm{bulk}}^{e}+U_{\mathrm{bulk}}^{o}=2 \times \int_{0}^{\infty} d k \frac{k}{2 \pi} \frac{1}{e^{k / T}-1} .
$$

Hence $U_{\text {bulk }}=\pi T^{2} / 6 . U_{\text {imp }}$ is the piece from the even sector that scales as $L^{-1}$. Hence

$$
U_{\mathrm{imp}}(T)=\frac{4 \Delta^{2}}{L} \int_{0}^{\infty} d k \frac{k}{e^{k / T}-1} \frac{k^{2}+\omega_{0}^{2}}{\left(k^{2}-\omega_{0}^{2}\right)^{2}+\left(4 \pi \Delta^{2} k\right)^{2}} .
$$

In the limit $\Delta \rightarrow 0$, the photons are decoupled from the impurity, and one expects $L U_{\mathrm{imp}}$ to be just the energy of a decoupled harmonic oscillator:

$$
\lim _{\Delta \rightarrow 0} L U_{\mathrm{imp}}=T^{2} \partial_{T} \log \left(\sum_{n=0}^{\infty} e^{-n \omega_{0} / T}\right)=\omega_{0} \frac{1}{e^{\omega_{0} / T}-1} .
$$

We have set the ground state energy of the harmonic oscillator to zero since in the scattering theory description the vacuum is defined to have zero energy. One can confirm that (4.17) has the proper zero coupling limit, using the identity:

$$
\lim _{\Delta^{2} / \omega_{0} \rightarrow 0} \frac{1}{\left(k^{2}-\omega_{0}^{2}\right)^{2}+\left(4 \pi \Delta^{2} k\right)^{2}}=\frac{1}{8 \Delta^{2} \omega_{0}^{2}} \delta\left(k-\omega_{0}\right) .
$$

Then,

$$
\lim _{\Delta \rightarrow 0} L U_{\mathrm{imp}}(T)=\omega_{0} \frac{1}{e^{\omega_{0} / T}-1}
$$

which agrees with (4.18). 
In common physical situations, $\omega_{0}^{2} \gg\left(4 \pi \Delta^{2}\right)^{2}$. Making this approximation in (4.17), one can extract the following low and high temperature behaviours:

$$
\begin{aligned}
& U_{\mathrm{imp}}(T) \approx \frac{2 \pi^{2}}{3} \frac{\Delta^{2}}{L} \frac{T^{2}}{\omega_{0}^{2}}, \quad T \ll \omega_{0} ; \\
& U_{\mathrm{imp}}(T) \approx \frac{T}{L}, \quad T \gg \omega_{0} .
\end{aligned}
$$

\section{Computation of Correlators}

In this section we will consider how to calculate several types of correlators that are useful in quantum optics problems. We begin with time-ordered correlators.

\subsection{Time-Ordered Correlators}

There are two possible pictures in which to view the defect. The first, as in section 3 , treats the defect as a point in space. Particles then scatter to and from the defect. Because the Hamiltonian of the theory is altered by the defect on any constant time surface, the particle spectrum is altered. This is reflected in the linear relations between the particle creation/destruction operators as in 3.1 and 3.2. In the second picture the defect is a point in time, an initial condition. Away from the defect the Hamiltonian of the theory remains unchanged and so the particle spectrum in the theory is the same. This is of particular advantage in calculating correlators. The defect, as an operator, is expressible in terms of the unperturbed Hilbert space and the correlator is then calculated by inserting the defect within the free fields forming the correlator in a time-ordered fashion:

$$
\left\langle T\left(O_{1}\left(x_{1}\right) \cdots O_{n}\left(x_{n}\right)\right)\right\rangle=\frac{\left\langle T\left(O_{1}^{\text {free }}\left(x_{1}\right) \cdots \widetilde{\mathbf{D}} O_{n}^{\text {free }}\left(x_{n}\right)\right)\right\rangle}{\langle\widetilde{\mathbf{D}}\rangle} .
$$

On the l.h.s. of the above equation, the fields are the full Heisenberg fields whereas the time evolution of the fields on the r.h.s. is governed by the free Hamiltonian. $\widetilde{\mathbf{D}}$ (to be distinguished from the $\mathbf{D}$ of section 3) then is no more than the S-matrix for the theory. It has a particularly simple form

$$
\widetilde{\mathbf{D}}=\exp \left[\int_{-\infty}^{\infty} d k \widetilde{T}(k) A_{+}^{\dagger}(k) A_{-}(k)+\int_{0}^{\infty} \widetilde{R}(k)\left(A_{+}^{\dagger}(k) A_{+}^{\dagger}(-k)+A_{-}(k) A_{-}(-k)\right)\right] .
$$

The \pm subscripts on the photon operators indicate on which side of the defect the particle lives: + for $t>0$ and - for $t<0$. The first term in the exponential transmits particles through the defect, destroying a - particle and replacing it with its + counterpart. 
Likewise the latter two terms represent reflections off the defect. Given $\widetilde{\mathbf{D}}$ 's form, it is completely represented in terms of one particle scattering amplitudes. This is a consequence of the theory's integrability. The form of the terms in the exponential are constrained by momentum conservation; the total momentum must sum to zero. Because all the terms in the exponential commute with one another, there are no ordering problems in representing $\widetilde{\mathbf{D}}$. We have assumed here that $\langle 0|\widetilde{\mathbf{D}}| 0\rangle=1$, that is the defect entropy is zero. With finite temperature this will not the case.

To distinguish between the two pictures, space-time coordinates in the first picture (the physical picture with the defect as a boundary condition) will be written as $(x, t)$ and the space-time coordinates in the second picture (with the defect as an initial condition) by $(\widehat{x}, \widehat{t})$. The two are then related by $(t, x)=(\widehat{x}, \widehat{t})$. To compute the elements of $\widetilde{\mathbf{D}}, \widetilde{T}(k)$ and $\widetilde{R}(k)$, one first expresses the action in the rotated picture, obtained from 3.3 via a Euclidean rotation and a reflection: $x \rightarrow \widehat{t}$ and $t \rightarrow \widehat{x}$. As before we can then derive an equation of motion with the $S^{ \pm}$eliminated:

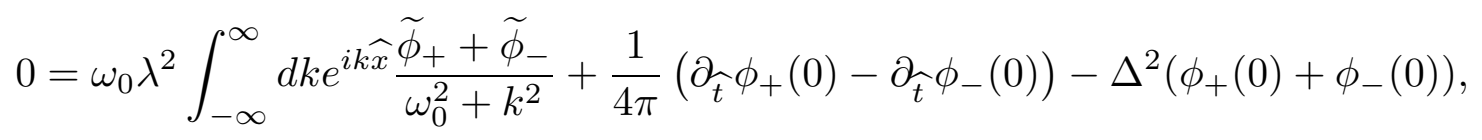

where $\phi_{+}$and $\phi_{-}$are defined via $\phi=\theta(\widehat{t}) \phi_{+}+\theta(-\widehat{t}) \phi_{-}$. As in section 3 , we have a continuity equation,

$$
\phi_{+}(\widehat{t}=0)=\phi_{-}(\widehat{t}=0) .
$$

To interpret the action of the above two equations on $\widetilde{\mathbf{D}}$, we divide each into a part involving $\phi_{+}$and a part involving $\phi_{-}$. So both have the form $f_{+}\left(\phi_{+}\right)-f_{-}\left(\phi_{-}\right)=0$. In terms of $\widetilde{\mathbf{D}}$ these become

$$
f_{+}\left(\phi_{+}\right) \widetilde{\mathbf{D}} g\left(\phi_{-}\right)|0\rangle=\langle 0| h\left(\phi_{+}\right) \widetilde{\mathbf{D}} f_{-}\left(\phi_{-}\right)
$$

$g\left(\phi_{-}\right)$and $h\left(\phi_{+}\right)$represent arbitrary insertions of the fields $\phi_{-}$and $\phi_{+}$between $\widetilde{\mathbf{D}}$ and the vacuum state. (5.5) tells us that as the fields $f_{+}\left(\phi_{+}\right)$cross the defect $\widetilde{\mathbf{D}}$, they are transformed into $f_{-}\left(\phi_{-}\right)$.

To determine the form of $\widetilde{T}(k)$ and $\widetilde{R}(k)$, we expand out $\widetilde{\mathbf{D}}$ to first order in $\widetilde{T}(k)$ and $\widetilde{R}(k)$ and substitute into the above two equations. Consistency at the one particle level then yields

$$
\begin{aligned}
& \widetilde{T}(k)=\frac{\omega_{0}^{2}+k^{2}}{\omega_{0}^{2}+k^{2}+4 \pi \Delta^{2}|k|} \\
& \widetilde{R}(k)=\frac{-4 \pi \Delta^{2}|k|}{\omega_{0}^{2}+k^{2}+4 \pi \Delta^{2}|k|}
\end{aligned}
$$


$\widetilde{T}(k)$ and $\widetilde{R}(k)$ can be related to the scattering matrices of section 3 via an analytic continuation. We see

$$
\widetilde{T}(k)(|k| \rightarrow-i k, k \rightarrow i|k|)=T(k),
$$

and similarly with $\widetilde{R}(k)$. This analytic continuation is effectively a rotation of the defect back to the t-axis. Here energies and momenta are continued via $E \rightarrow-i p$ and $p \rightarrow i E$.

Knowing $\widetilde{\mathbf{D}}$ we can now go on to calculate correlators. We will compute the two-point function of the photon field. We have for this correlator,

$$
\begin{aligned}
\left\langle T\left(\phi(\widehat{x}, \widehat{t}) \phi\left(\widehat{x}^{\prime}, \widehat{t}^{\prime}\right)\right)\right\rangle=\theta\left(\widehat{t}-\widehat{t}^{\prime}\right)\left[\theta(\widehat{t}) \theta\left(\widehat{t}^{\prime}\right)\left\langle\phi(\widehat{x}, \widehat{t}) \phi\left(\widehat{x}^{\prime}, \widehat{t}^{\prime}\right) \widetilde{\mathbf{D}}\right\rangle\right. & +\theta(\widehat{t}) \theta\left(-\widehat{t}^{\prime}\right)\left\langle\phi(\widehat{x}, \widehat{t}) \widetilde{\mathbf{D}} \phi\left(\widehat{x}^{\prime}, \widehat{t}^{\prime}\right)\right\rangle \\
& \left.+\theta(\widehat{t}) \theta\left(-\widehat{t}^{\prime}\right)\left\langle\widetilde{\mathbf{D}} \phi(\widehat{x}, \widehat{t}) \phi\left(\widehat{x}^{\prime}, \widehat{t}^{\prime}\right)\right\rangle\right] \\
& +\left(\widehat{t} \leftrightarrow \widehat{t}^{\prime}, \widehat{x} \leftrightarrow \widehat{x}^{\prime}\right)
\end{aligned}
$$

Each of the pieces of $\langle T(\phi \phi)\rangle$ is easily calculated as $\phi \phi$ only couples to the two particle contribution to $\widetilde{\mathbf{D}}$. So

$$
\begin{aligned}
& \left\langle\phi(\widehat{x}, \widehat{t}) \phi\left(\widehat{x}^{\prime}, \widehat{t}^{\prime}\right) \widetilde{\mathbf{D}}\right\rangle=\left\langle\phi(\widehat{x}, \widehat{t}) \phi\left(\widehat{x}^{\prime}, \widehat{t}^{\prime}\right)\right\rangle_{\text {free }}+2 \int_{0}^{\infty} d k \frac{\widetilde{R}(k)}{k} e^{-\left(\widehat{t}+\widehat{t}^{\prime}\right)|k|} \cos \left(k\left(\widehat{x}-\widehat{x}^{\prime}\right)\right) \\
& \left\langle\phi(\widehat{x}, \widehat{t}) \widetilde{\mathbf{D}} \phi\left(\widehat{x}^{\prime}, \widehat{t}^{\prime}\right)\right\rangle=\int_{-\infty}^{\infty} d k \frac{\widetilde{T}(k)}{|k|} e^{\left.-\widehat{t}-\widehat{t}^{\prime}\right)|k|+i k\left(\widehat{x}-\widehat{x}^{\prime}\right)} \\
& \left\langle\widetilde{\mathbf{D}} \phi(\widehat{x}, \widehat{t}) \phi\left(\widehat{x}^{\prime}, \widehat{t}^{\prime}\right)\right\rangle=\left\langle\phi(\widehat{x}, \widehat{t}) \phi\left(\widehat{x}^{\prime}, \widehat{t}^{\prime}\right)\right\rangle_{\text {free }}+2 \int_{0}^{\infty} d k \frac{\widetilde{R}(k)}{k} e^{\left(\widehat{t}+\widehat{t}^{\prime}\right)|k|} \cos \left(k\left(\widehat{x}-\widehat{x}^{\prime}\right)\right) .
\end{aligned}
$$

The correlator $\langle T(\phi \phi)\rangle$ has now been calculated in the picture with the defect as initial condition. To obtain this same correlator in the physical picture, we rotate and reflect the system back via $\widehat{x} \rightarrow t, \widehat{t} \rightarrow x$. If, for example, we supposed $x=x^{\prime}>0$ in the original system, the correlator becomes

$$
\begin{aligned}
\left\langle T\left(\phi(x, t) \phi\left(x, t^{\prime}\right)\right\rangle\right. & =\left\langle\phi(\widehat{x}, \widehat{t}) \phi\left(\widehat{x}^{\prime}, \widehat{t}\right) \widetilde{\mathbf{D}}\right\rangle \\
& =\left\langle\phi(t, x) \phi\left(t^{\prime}, x\right)\right\rangle_{\text {free }}+2 \int_{0}^{\infty} \frac{\widetilde{R}(k)}{k} e^{-2 x|k|} \cos \left(k\left(t-t^{\prime}\right)\right) .
\end{aligned}
$$

The correlator only depends on $\widetilde{R}(k)$ as we have assumed that both space points are on the same side of the defect. 


\subsection{Non-Time Ordered Correlators}

So far we have only considered time-ordered correlators. But correlators not so ordered can also be easily computed, a good thing as the correlators in which one is often interested for quantum optic calculations are not time-ordered. For example, it common to want to compute Glauber correlators, correlators of normal ordered products of the creation and destruction pieces of the photon fields. In this section we will consider three examples of non-time ordered correlators: the photon density in the fiber at finite temperature, the spontaneous emission spectrum for an excited oscillator state, and the correlators of the defect degrees of freedom. We will do these calculations in a finite temperature formalism. It turns out the calculations are easier to do in such a framework. Euclidean correlators which are not time ordered are not convergent for all of spacetime. This makes the rotation of the defect between viewing it as a boundary condition and viewing it as an initial condition ill-defined for such correlators. But in a finite temperature formalism, unordered correlators can be related to time-ordered temperature correlators for which the rotation, $(x, t) \leftrightarrow(\widehat{x}, \widehat{t})$, poses no difficulties.

Temperature correlators are computed in much the same manner as the time-ordered correlators of the previous section. The primary difference lies in the mode expansions. As we are at finite temperature, $\mathrm{T}$, we expand the fields on a cylinder of circumference $\beta=1 / T=4 \pi l$ :

$$
\begin{aligned}
\phi_{l} & =\sum_{n \geq 1} \frac{1}{n^{1 / 2}}\left(\phi_{n} e^{-n \widehat{z} / l}+\phi_{-n} e^{n \widehat{z} / l}\right), \quad \widehat{z}=(\widehat{t}+i \widehat{x}) / 2 \\
\phi_{r} & =\sum_{n \geq 1} \frac{1}{n^{1 / 2}}\left(\bar{\phi}_{n} e^{-n \widehat{\bar{z}} / l}+\bar{\phi}_{-n} e^{n \widehat{\bar{z}} / l}\right), \quad \widehat{\bar{z}}=(\widehat{t}-i \widehat{x}) / 2 \\
\phi & =\phi_{l}+\phi_{r} .
\end{aligned}
$$

We have divided $\phi$ into its left and right moving components. Here the mode expansions are periodic in $\widehat{x}$ (and not $\widehat{t}$ ) as we intend to eventually interchange time and space (so as to return to the original system).

The defect operator $\widetilde{\mathbf{D}}$ for $T \neq 0$ is constructed in exactly the same fashion as in the $T=0$ case. We substitute the mode expansions into the equations of motion and find

$$
\begin{aligned}
0 & =\frac{1}{4 \pi}\left(\partial_{t} \phi_{+}-\partial_{t} \phi_{-}\right)-\Delta^{2}\left(\phi_{+}+\phi_{-}\right)+\lambda\left(S^{+}+S^{-}\right) \\
S^{ \pm}(\widehat{x}) & =\lambda \sum_{n \geq 1} \frac{1}{n^{1 / 2}}\left[\frac{e^{i \omega_{n} \widehat{x}}}{\omega_{0} \mp i \omega_{n}}\left(\phi_{-n}+\bar{\phi}_{n}\right)+\frac{e^{-i \omega_{n} \widehat{x}}}{\omega_{0} \pm i \omega_{n}}\left(\phi_{n}+\bar{\phi}_{-n}\right)\right] .
\end{aligned}
$$


where the Matsubara frequencies are given by $\omega_{n}=n / 2 l$. From this we construct $\widetilde{\mathbf{D}}$ as before:

$$
\widetilde{\mathbf{D}}=g \exp \left[\sum_{n \geq 1} \widetilde{T}\left(\omega_{n}\right)\left(\phi_{-n}^{+} \phi_{n}^{-}+\bar{\phi}_{-n}^{+} \bar{\phi}_{-n}^{-}\right)+\widetilde{R}\left(\omega_{n}\right)\left(\phi_{-n}^{+} \bar{\phi}_{-n}^{+}+\phi_{n}^{-} \bar{\phi}_{n}^{-}\right)\right]
$$

where $\widetilde{T}$ and $\widetilde{R}$ are as in (5.6). In the $T=0$ case we were able to set $g$, the boundary entropy to 1 . Here we cannot do so. The partition function of the system is

$$
Z=\left\langle 0\left|e^{\widehat{t}_{1} H} \widetilde{\mathbf{D}} e^{\widehat{t}_{2} H}\right| 0\right\rangle
$$

where time extends to some $\widehat{t}_{1}$ and $\widehat{t}_{2}$ on either side of the defect. $\left(\widehat{t}_{1}+\widehat{t}_{2}\right.$ thus defines the length of the cylinder which we are on.) As $\widehat{t}_{1}, \widehat{t}_{2} \rightarrow 0$, we expect $\mathrm{Z}$ to reduce to its defect contribution. Thus

$$
Z_{i m p}=\lim _{\widehat{t}_{1}, \widehat{t}_{2} \rightarrow 0} Z=\langle 0|\widetilde{\mathbf{D}}| 0\rangle=g
$$

Our TBA calculation from the previous section gives us

$$
\log g=-4 \Delta^{2} \int_{0}^{\infty} d k \frac{\left(\omega_{0}^{2}+k^{2}\right) \log \left(1-e^{-k / T}\right)}{\left(\omega_{0}^{2}-k^{2}\right)^{2}+16 \pi^{2} \Delta^{4} k^{2}}
$$

We point out that knowledge of $g$ is not necessary for the computation of correlators as it is a constant which is always normalized away.

We now go on to compute the number of photons, $n(\omega)$, in the fiber. $n(\omega)$ is given in terms of the two point photon correlator:

$$
n(\omega)=\frac{\omega}{2 \pi} \int d t e^{-i \omega t}\left\langle\phi^{c}(x, t) \phi^{d}(x, 0)\right\rangle,
$$

where $\phi^{c / d}$ are the creation/destruction pieces of the field. These pieces are defined for non-free field theories to be (see [9])

$$
\phi^{c / d}(x, t)= \pm \frac{1}{2 \pi i} \int d t^{\prime} \frac{\phi\left(x, t^{\prime}\right)}{t^{\prime}-t \mp i \epsilon}
$$

The factor $\omega / 2 \pi$ in (5.17) serves to remove the relativistic normalization present in the mode expansion of the photon fields. $\left\langle\phi^{c} \phi^{d}\right\rangle$ is not time ordered and as indicated previously 
would be difficult to compute directly. However by the fluctuation-dissipation theorem we can reexpress this correlator in terms of a retarded Green's function:

$$
\begin{aligned}
\int d t e^{-i \omega t}\left\langle\phi^{c}(x, t) \phi^{d}(x, 0)\right\rangle & =\frac{2}{e^{\beta \omega}-1} \operatorname{Im} G_{R}(-\omega) \theta(\omega) ; \\
G_{R}(\omega) & =-i \int_{0}^{\infty} d t e^{i \omega t}\langle[\phi(x, t), \phi(x, 0)]\rangle .
\end{aligned}
$$

This sort of identity between correlators is easily proven by an explicit evaluation of the thermal trace together with an insertion of states between the operators (see [10]). The advantage of writing $n(\omega)$ in this form is that $G_{R}(\omega)$ is available directly as the analytic continuation of a temperature Green's function,

$$
G_{R}(\omega)=G_{T}(-i \omega+\epsilon)=-\left.\int_{0}^{\beta} e^{i \omega_{n} t}\langle\phi(x, t) \phi(x, 0)\rangle_{T}\right|_{\omega_{n}=-i \omega+\epsilon}
$$

and $G_{T}$ is exactly what we are set up to calculate.

To compute $G_{T}=-\langle\phi(x, t) \phi(x, 0)\rangle_{T}$ we go into the rotated system. Assuming $x>0$ we have

$$
\begin{aligned}
G_{T} & =-\langle\phi(x, t) \phi(x, 0)\rangle_{t}=-\left\langle\phi_{+}(\widehat{x}, \widehat{t}) \phi_{+}(\widehat{x}, 0) \widetilde{\mathbf{D}}\right\rangle \\
& =-\frac{1}{l} \sum_{n \geq 1} \frac{1}{\omega_{n}} \cos \left(\omega_{n} t\right)\left[1+\widetilde{R}\left(\omega_{n}\right) e^{-2 \omega_{n} x}\right] .
\end{aligned}
$$

Following the prescription above, we then find for $n(\omega)$,

$$
\begin{aligned}
n(\omega)=\frac{2 \theta(\omega)}{e^{\beta \omega}-1}[1 & +\sin (2 \omega x) \frac{4 \pi \Delta^{2} \omega\left(\omega^{2}-\omega_{0}^{2}\right)}{\left(\omega^{2}-\omega_{0}^{2}\right)^{2}+16 \pi^{2} \Delta^{4} \omega^{2}} \\
& \left.-\cos (2 \omega x) \frac{16 \pi^{2} \Delta^{4} \omega^{2}}{\left(\omega^{2}-\omega_{0}^{2}\right)^{2}+16 \pi^{2} \Delta^{4} \omega^{2}}\right] .
\end{aligned}
$$

We see that we obtain the Planck distribution (times two - one for right moving photons and one for left moving photons) plus a correction term. This distribution should not be compared with the TBA calculation of the previous section. The distribution there is not that of pure photons but rather of their even and odd combinations that have been averaged over the length of the system.

We now move on to calculating the spontaneous emission spectrum, $P(\omega)$, arising from the decay of the oscillator from its first excited state. As the electric field is given in terms of $\phi$ by $E \propto \partial_{t} \phi$, we define the power spectrum to be

$$
\begin{aligned}
P(\omega) & =\frac{1}{4 \pi^{2}} \int d t d t^{\prime} e^{i \omega\left(t-t^{\prime}\right)}\left\langle S^{-}(0) \partial_{t} \phi^{c}(x, t) \partial_{t} \phi^{d}\left(x, t^{\prime}\right) S^{+}(0)\right\rangle \\
& =\frac{\omega^{2}}{4 \pi^{2}} \int d t d t^{\prime} e^{i \omega\left(t-t^{\prime}\right)}\left\langle S^{-}(0) \phi(x, t)\right\rangle\left\langle\phi\left(x, t^{\prime}\right) S^{+}(0)\right\rangle .
\end{aligned}
$$


In the second line the four-point correlator has first been factored into two two-point correlators and $\phi^{c / d}$ replaced with $\phi$, both possible as $S^{ \pm}$only creates or destroys single particles, and then the resulting expression has been integrated by parts. These steps are only possible at $T=0$. However we will let $T \neq 0$ in order to compute the two-point functions and then take $T \rightarrow 0$ at the end. We can make a further simplification by noting that

$$
\int d t e^{-i \omega t}\left\langle S^{-}(0) \phi(x, t)\right\rangle=\left[\int d t e^{i \omega t}\left\langle\phi(x, t) S^{+}(0)\right\rangle\right]^{*} .
$$

We thus need only to compute $\left\langle\phi(x, t) S^{+}(0)\right\rangle$.

As before we compute $\left\langle\phi(x, t) S^{+}(0)\right\rangle$ through a temperature correlator. In this case we have

$$
\begin{aligned}
\int \frac{d t}{2 \pi} e^{i \omega t}\left\langle\phi(x, t) S^{+}(0)\right\rangle & =\frac{i}{2 \pi} \frac{\theta(\omega)}{1-e^{-\beta \omega}}\left(G_{T}(-i \omega+\epsilon)-G_{T}(-i \omega-\epsilon)\right) \\
G_{T}\left(\omega_{n}\right) & =-\int_{0}^{\beta} d t e^{i \omega_{n} t}\left\langle\phi(x, t) S^{+}(0)\right\rangle_{T} .
\end{aligned}
$$

Our expression involves both the retarded $G_{T}(-\omega+\epsilon)$ and the advanced $G_{T}(-\omega-\epsilon)$ Green's functions as the combination $\phi S^{+}$is not Hermitean. Again this identity can be verified by evaluating the thermal trace and inserting a complete set of states between $\phi$ and $S^{+}$. The temperature correlator is computed as before by rotating the system. Using (5.12) we then find,

$$
\begin{aligned}
\left\langle\phi(x, t) S^{+}(0)\right\rangle_{T} & =\left\langle\phi_{+}(\widehat{x}, \widehat{t}) S^{+}(0) \widetilde{\mathbf{D}}\right\rangle \\
& =\frac{\lambda}{2 l} \sum_{n \geq 1} \frac{\widetilde{T}\left(\omega_{n}\right)}{\omega_{n}}\left[\frac{e^{-\omega_{n}(x+i t)}}{\omega_{0}-i \omega_{n}}-\frac{e^{-\omega_{n}(x-i t)}}{\omega_{0}+i \omega_{n}}\right] .
\end{aligned}
$$

The Matsubara decomposition of this correlator is

$$
G_{T}\left(\omega_{n}\right)=-\frac{2 \pi \lambda}{\omega_{n}} \frac{1}{\omega_{0}-i \omega_{n}}\left(\theta\left(\omega_{n}\right) e^{-\omega_{n} x} \widetilde{T}\left(\omega_{n}\right)-\theta\left(-\omega_{n}\right) e^{\omega_{n} x} \widetilde{T}\left(-\omega_{n}\right)\right)
$$

Putting everying thing together and taking $T \rightarrow 0$, we find the power spectrum to be

$$
\begin{aligned}
P(\omega)= & \frac{2 \Delta^{2} \omega_{0} \theta(\omega)}{\left(\omega_{0}-\omega\right)^{2}} \frac{\left(\omega_{0}^{2}-\omega^{2}\right)^{2}}{D}\left[1+\frac{\cos (2 \omega x)}{D}\left(\left(\omega_{0}^{2}-\omega^{2}\right)^{2}-16 \pi^{2} \Delta^{4} \omega^{2}\right)\right. \\
& \left.-\frac{\sin (2 \omega x)}{D}\left(8 \pi \Delta^{2} \omega\left(\omega_{0}^{2}-\omega^{2}\right)\right)\right], \\
D=\left(\omega_{0}^{2}-\omega^{2}\right)^{2}+16 \pi^{2} \Delta^{4} \omega^{2} &
\end{aligned}
$$


Typically the power spectra is computed for near resonance $\left(\omega \simeq \omega_{0}\right)$. Near resonance $P(\omega)$ reduces to

$$
P(\omega)=\theta(\omega) \frac{4 \Delta^{2} \omega_{0} \sin ^{2}(\omega x)}{\left(\omega_{0}-\omega\right)^{2}+4 \pi^{2} \Delta^{4}} .
$$

We see that $P(\omega)$ takes on the familiar Lorentzian lineshape with halfwidth equal to $4 \pi \Delta^{2}$. Near resonance the lowest order term of $P(\omega)$ has the form predicted by classical theory (see [11]) and the same form predicted by first order perturbation theory employing the master equation (see [7]). The spatial dependence in the power spectra is an artifact of not including the power from the magnetic field. Doing so leaves $P(\omega)$ independent of $x$.

This basic Lorentzian lineshape is also shared by a two-level system interacting with a photon field near resonance (see [7]). Where the physics of the the two-level system disagrees with that of an SHO defect is in the presence of an external driving field. For a $\mathrm{SHO}$ defect the response spectrum resulting from an external field is a $\delta$-function. This is only true for a two-level system for a weak driving field. As the driving field intensity is increased, the $\delta$-function spectrum of the two-level system broadens and then splits into three Lorentzians, one centered at the driving frequency and two sidebands equidistant from the central one. The two systems diverge in behaviour in large external fields as the two-level system saturates in its upper level for such field whereas the SHO can keep being excited into ever higher levels. At low driving intensities the SHO spends its time mostly its in ground state or its first excited level and so mimics a two-level system.

As the final illustration of computing non-time ordered correlators, we compute the correlators of the defect degrees of freedom, i.e. $\left\langle S^{\gamma}(t) S^{\delta}(0)\right\rangle, \gamma, \delta= \pm$. Even though the defect degrees of freedom have been integrated out, we can still compute their correlators as we know how $S^{ \pm}$are related to the photon field. As before $\left\langle S^{\gamma}(t) S^{\delta}(0)\right\rangle$ is related to a temperature correlator via

$$
\begin{aligned}
\left\langle S^{\gamma} S^{\delta}\right\rangle(\omega)=\int \frac{d t}{2 \pi} e^{i \omega t}\left\langle S^{\gamma}(t) S^{\delta}(0)\right\rangle & =\frac{i}{2 \pi} \frac{\theta(\omega)}{1-e^{-\beta \omega}}\left(G_{T}(-i \omega+\epsilon)-G_{T}(-i \omega-\epsilon)\right) \\
G_{T}\left(\omega_{n}\right) & =-\int_{0}^{\beta} d t e^{i \omega_{n} t}\left\langle S^{\gamma}(t) S^{\delta}(0)\right\rangle_{T}
\end{aligned}
$$

$\left\langle S^{\gamma}(t) S^{\delta}(0)\right\rangle$ is computed by rotating the system

$$
\left\langle S^{\gamma}(t) S^{\delta}(0)\right\rangle_{T}=\left\langle S_{+}^{\gamma}(\widehat{x}) \widetilde{\mathbf{D}} S_{-}^{\delta}(0)\right\rangle_{T}
$$

As $S^{\gamma}$ and $S^{\delta}$ are defined at $\widehat{x}=0$, we have a choice as to their ordering with respect to $\widetilde{\mathrm{D}}$. The expressions for the correlators are independent of this choice (as they should be). 
We have chosen in the above to place $S^{\gamma}$ and $S^{\delta}$ on either side of $\widetilde{\mathbf{D}}$. Using (5.12), the correlator on the r.h.s. of the above is easily calculated:

$$
\left\langle S_{+}^{\gamma}(\widehat{x}) \widetilde{\mathbf{D}} S_{-}^{\delta}(0)\right\rangle_{T}=\Delta^{2} \omega_{0} \sum_{n \geq 1} \frac{1}{n}\left[\frac{e^{i \omega_{n} t}}{\left(\omega_{0}-i \gamma \omega_{n}\right)\left(\omega_{0}+i \delta \omega_{n}\right)}+\frac{e^{-i \omega_{n} t}}{\left(\omega_{0}+i \gamma \omega_{n}\right)\left(\omega_{0}-i \delta \omega_{n}\right)}\right] .
$$

Then by $(5.30),\left\langle S^{\gamma} S^{\delta}\right\rangle(\omega)$ equals

$$
\left\langle S^{\gamma} S^{\delta}\right\rangle(\omega)=\frac{2 \Delta^{2} \omega_{0} \theta(\omega)}{\omega\left(1-e^{-\beta \omega}\right)} \frac{\left(\omega_{0}^{2}-\omega^{2}\right)^{2}}{\left(\omega_{0}+\gamma \omega\right)\left(\omega_{0}-\delta \omega\right)} \frac{1}{\left(\omega_{0}^{2}-\omega^{2}\right)^{2}+16 \pi^{2} \Delta^{4} \omega^{2}} .
$$

One should notice that the correlators $\left\langle S^{+} S^{-}\right\rangle,\left\langle S^{+} S^{+}\right\rangle$, and $\left\langle S^{-} S^{-}\right\rangle$do not vanish. This is a result of the coupling between the photon field and oscillator. Because the interaction is $\left(S^{+}+S^{-}\right) \phi\left(\right.$ and not $\left.S^{+} \phi^{d}+S^{-} \phi^{c}\right), S^{+}$and $S^{-}$have both creation and destruction parts and so undergo vacuum fluctuations.

\section{Multiple Defects}

The analysis of a single defect in the previous sections can be easily extended to multiple defects. We will first consider a set of $n$ defects placed arbitrarily and then move on to a periodic array of defects.

\section{1. $n$ Defects}

The action for a set of $n$ defects is

$$
\begin{aligned}
S=\int d t d x & {\left[\frac{1}{8 \pi}\left(\left(\partial_{t} \phi\right)^{2}+\left(\partial_{x} \phi\right)^{2}\right)\right.} \\
+ & \left.\sum_{i=1}^{n} \delta\left(x-x_{i}\right)\left(-S_{i}^{-} \partial_{t} S_{i}^{+}+\omega_{i} S_{i}^{+} S_{i}^{-}-\lambda_{i}\left(S_{i}^{-}+S_{i}^{+}\right) \phi+\Delta_{i}^{2} \phi^{2}\right)\right],
\end{aligned}
$$

where the i-th defect has been placed at $x_{i}, x_{i+1}>x_{i}>x_{i-1}$. We have allowed each defect to have different coupling strengths to the photon field. The equations of motion for this action are

$$
\begin{aligned}
& 0=\sum_{i=1}^{n} \delta\left(x-x_{i}\right)\left[\lambda_{i}\left(S_{i}^{-}+S_{i}^{+}\right)-2 \Delta_{i}^{2} \phi\right]+\frac{1}{4 \pi}\left(\partial_{t}^{2} \phi+\partial_{x}^{2} \phi\right) ; \\
& 0=\delta\left(x-x_{i}\right)\left(-\lambda_{i} \phi+\omega_{i} S_{i}^{ \pm} \mp \partial_{t} S_{i}^{ \pm}\right) .
\end{aligned}
$$


By assuming $\phi$ has a free solution in the intervals in between the defects,

$$
\begin{gathered}
\phi=\phi_{n+1} \theta\left(x-x_{n}\right)+\phi_{n} \theta\left(x_{n}-x\right) \theta\left(x-x_{n-1}\right)+\cdots \\
\quad+\phi_{2} \theta\left(x_{2}-x\right) \theta\left(x-x_{1}\right)+\phi_{1} \theta\left(x_{1}-x\right), \\
0=\left(\partial_{t}^{2}+\partial_{x}^{2}\right) \phi_{i}, \quad 1 \leq i \leq n+1,
\end{gathered}
$$

the above equations of motion can be reduced to

$$
\begin{aligned}
0=\sum_{i=1}^{n} \delta\left(x-x_{i}\right)\left[\omega_{i} \lambda_{i}^{2} \int_{-\infty}^{\infty} d k e^{k t}\right. & \frac{\widetilde{\phi}_{i+1}(k)+\widetilde{\phi}_{i}(k)}{k^{2}-\omega_{i}^{2}} \\
& \left.+\Delta_{i}^{2}\left(\phi_{i+1}+\phi_{i}\right)-\frac{1}{4 \pi}\left(\partial_{x} \phi_{i+1}-\partial_{x} \phi_{i}\right)\right],
\end{aligned}
$$

where $\widetilde{\phi}_{i}$ is, as before,

$$
\phi_{i}\left(x_{i}, t\right)=\int_{-\infty}^{\infty} d k e^{k t} \widetilde{\phi}\left(k, x_{i}\right) .
$$

We have thus obtained a set of $n$ uncoupled equations. These can be solved in same fashion as in Section 3.

Let $\mathbf{D}_{i}$ be the defect operator for the i-th defect. We define the transmission and reflection matrices across this defect by

$$
\mathbf{D}_{i} A_{i+1}(k)=T_{i} A_{i}(k) \mathbf{D}_{i}+R_{i}(k) \mathbf{D}_{i} A_{i+1}(-k),
$$

where the $A_{i}(k)$ 's arise from the mode expansion of $\phi_{i}$. (6.4) then fixes $T_{i}$ and $R_{i}$ to be

$$
\begin{aligned}
& T_{i}(k)=T\left(k, \omega_{i}, \Delta_{i}\right), \\
& R_{i}(k)=e^{-2 i k x_{i}} R\left(k, \omega_{i}, \Delta_{i}\right),
\end{aligned}
$$

where $T\left(k, \omega_{i}, \Delta_{i}\right)$ and $R\left(k, \omega_{i}, \Delta_{i}\right)$ are the matrices in (3.12). We see that $R_{i}$ has picked up a phase, relative to $R$, a result of a reflection away from $x=0$.

$T_{i}$ and $R_{i}$ describe the transmission and reflection across the defect at $x_{i}$. However the transmission and reflection amplitudes across all $n$ defects is considerably more complicated; one has to consider multiple reflections and transmissions as the photon traverses the set of defects. To simplify the computation, we introduce a transmission matrix defined via

$$
\left(\begin{array}{c}
A_{i+1}(k) \\
A_{i+1}(-k)
\end{array}\right)=\mathbf{M}_{i}\left(\begin{array}{r}
A_{i}(k) \\
A_{i}(-k)
\end{array}\right) .
$$


Using (6.6) and its conjugate,

$$
A_{i}(-k) \mathbf{D}_{i}=T_{i}(k) \mathbf{D}_{i} A_{i+1}(-k)+R_{i}(k) A_{i}(k) \mathbf{D}_{i},
$$

$\mathbf{M}_{i}$ is found to be

$$
\mathbf{M}_{i}=\left[\begin{array}{ll}
T_{i}^{-1}(-k) & R_{i}(k) T_{i}^{-1}(k) \\
R_{i}(-k) T_{i}^{-1}(-k) & T_{i}^{-1}(k)
\end{array}\right] .
$$

We can then write down the transmission matrix across all the defects:

$$
\mathbf{M}_{\mathbf{T}}=\mathbf{M}_{n} \cdots \mathbf{M}_{1}
$$

The overall scattering amplitudes, $T_{T}$ and $R_{T}$ are defined by

$$
\mathbf{D}_{\mathbf{T}} A_{n+1}(k)=T_{T} A_{1}(k) \mathbf{D}_{\mathbf{T}}+R_{T}(k) \mathbf{D}_{\mathbf{T}} A_{n+1}(-k),
$$

where $\mathbf{D}_{\mathbf{T}}=\mathbf{D}_{n} \cdots \mathbf{D}_{1}$. These amplitudes are then related to $\mathbf{M}_{\mathbf{T}}$ via

$$
\mathbf{M}_{\mathbf{T}}=\left[\begin{array}{ll}
T_{T}^{-1}(-k) & R_{T}(k) T_{T}^{-1}(k) \\
R_{T}(-k) T_{T}^{-1}(-k) & T_{T}^{-1}(k)
\end{array}\right]
$$

As it stands $\mathbf{M}_{\mathbf{T}}$ is not computable analytically (though certainly numerically) for an arbitrary set of $\mathrm{n} x_{i}$ 's, $\Delta_{i}$ 's, and $\omega_{i}$ 's. As such we will only consider a particularly simple case: the case of two defects. Taking $x_{1}=0, T_{T}(k)$ and $R_{T}(k)$ can the be computed to be

$$
\begin{aligned}
& T_{T}(k)=\frac{T_{1}(k) T_{2}(k)}{1-R_{1}(k) R_{2}(k)}=T_{1}(k) T_{2}(k) \sum_{n=0}^{\infty}\left(R_{1}(k) R_{2}(k)\right)^{n} \\
& R_{T}(k)=R_{2}(k)+\frac{R_{1}(k) T_{2}^{2}(k)}{1-R_{1}(k) R_{2}(k)}=R_{2}(k)+R_{1}(k) T_{2}^{2}(k) \sum_{n=0}^{\infty}\left(R_{1}(k) R_{2}(k)\right)^{n} .
\end{aligned}
$$

The latter equality in the above two equations shows that $T_{T}$ and $R_{T}$ can be both computed through adding up multiple reflections and transmissions as if this problem was a two interface problem in classical optics.

As with $T(k)$ and $R(k)$ in Section 3 , both $T_{T}$ and $R_{T}$ exhibit poles as a result of the oscillator defects. $R_{T}$ has simple poles at

$$
k_{p}= \pm \omega_{2}\left(1-\frac{4 \pi^{2} \Delta_{2}^{4}}{\omega_{2}^{2}}\right)^{1 / 2}-i 2 \pi \Delta_{2}^{2},
$$


indicative of reflection off the second defect. There is no similar pole for the first defect as we are scattering from the right. If we were scattering from the left, a pole in the variables $\omega_{1}$ and $\Delta_{1}$ would appear in $R_{T} . R_{T}$ and $T_{T}$ have another set of poles dependent upon $x_{2}$ representing a resonance arising from interactions between the two defects. In the limit $x_{2}=0$ and $\left(\Delta_{2}, \omega_{2}\right)=\left(\Delta_{1}, \omega_{1}\right)$, these poles appear at

$$
k_{p}= \pm \omega_{1}\left(1-\frac{16 \pi^{2} \Delta_{1}^{4}}{\omega_{1}^{2}}\right)^{1 / 2}-i 4 \pi \Delta_{1}^{2}
$$

In this case, the inverse lifetime has doubled, being the sum of the inverse lifetimes of the two oscillators considered separately. It sums for if either of the two oscillators emits a photon, the excited state will decay.

\subsection{Band Structure}

In this section we consider a periodic array of defects. With such a periodic structure, we expect the photon spectrum to be described by a band structure. Rather than compute the band structure by extracting it from $\mathbf{M}_{\mathbf{T}}$, we will be able to compute it through Bloch's theorem.

We begin with the Hamiltonian describing a three dimensional array of defects in the fiber and their interaction with a photon field:

$$
H=H_{\text {field }}+\sum_{i, j, k}\left(\omega_{0} S_{i j k}^{+} S_{i j k}^{-}+\lambda\left(S_{i j k}^{-}+S_{i j k}^{+}\right) \phi\left(x_{i}, 0,0\right)+\Delta^{2} \phi^{2}\left(x_{i}, 0,0\right)\right)
$$

Here $\{i, j, k\}$ marks out the locations of the defects in the array. This array is three dimensional. However the problem is still one dimensional as $\phi$, as before, does not depend on the transverse coordinates, $y$ and $z$. To reduce this Hamiltonian to one dimension, we define a collective oscillator representing a sum of all the oscillators in a plane at $x_{i}$ transverse to the direction of the fiber,

$$
S_{i}^{ \pm}=\frac{1}{\sqrt{N}} \sum_{j k} S_{i j k}^{ \pm}
$$

where $\mathrm{N}$ is the number of oscillators in this plane. $S_{i}^{ \pm}$has the expected commutator

$$
\left[S_{i}^{-}, S_{i}^{+}\right]=1
$$


With this commutation relation, the above Hamiltonian can be recast as

$$
H=H_{\text {field }}+\sum_{i=-\infty}^{\infty}\left(\omega_{0} S_{i}^{+} S_{i}^{-}-\sqrt{N} \lambda\left(S_{i}^{-}+S_{i}^{+}\right) \phi(i a)+N \Delta^{2} \phi^{2}(i a)\right)
$$

where $a$ is the lattice spacing of the array along the fiber. (We do not need to assume the lattice spacing in the planes transverse to the fiber is also a.) We see both couplings have been rescaled, $\lambda$ by $\sqrt{N}$ and $\Delta^{2}$ by $N$. The corresponding Minkowski action is

$$
\begin{aligned}
S= & \int d t d x\left[\frac{1}{8 \pi}\left(\left(\partial_{t} \phi\right)^{2}-\left(\partial_{x} \phi\right)^{2}\right)\right. \\
& \left.+\sum_{i=-\infty}^{\infty} \delta\left(x-x_{i}\right)\left(-i S_{i}^{-} \partial_{t} S_{i}^{+}-\omega_{0} S_{i}^{+} S_{i}^{-}+\sqrt{N} \lambda\left(S_{i}^{-}+S_{i}^{+}\right) \phi-N \Delta^{2} \phi^{2}\right)\right] .
\end{aligned}
$$

For a lattice spacing in the transverse planes of $10^{-7} \mathrm{~cm}$ and $D \sim 10^{-4} \mathrm{~cm}^{2}$, we have $\lambda / \omega_{0} \sim 10^{-2}$. This is large enough to produce observable results.

From (6.21) we can immediately write down the equations of motion governing such a periodic array of defects:

$$
\begin{aligned}
0=\sum_{n=-\infty}^{\infty} & {\left[\omega_{0} N \lambda^{2} \int_{-\infty}^{\infty} d k e^{i k t} \frac{\widetilde{\phi}_{n}(k, n a)+\widetilde{\phi}_{n-1}(k,(n-1) a)}{\omega_{0}^{2}-k^{2}}\right.} \\
& \left.-N \Delta^{2}\left(\phi_{n}(n a)+\phi_{n-1}((n-1) a)\right)+\frac{1}{4 \pi}\left(\partial_{x} \phi_{n}(n a)-\partial_{x} \phi_{(n-1)}(n a)\right)\right],
\end{aligned}
$$

where $a$ is the spacing between the defects. We have written $\phi$ as

$$
\phi(x)=\sum_{n=-\infty}^{\infty} \phi_{n}(x) \theta(n a-x) \theta(x-(n-1) a) .
$$

Each $\phi_{n}$ is free and so satisfies $\left(\partial_{t}^{2}-\partial_{x}^{2}\right) \phi_{n}=0$.

To solve this equation it is necessary to uncouple the various $\phi_{n}$ 's from one another. We do this via Bloch's theorem. It tells us the solutions to these equations must satisfy

$$
\phi_{n}(n a, t)=e^{i p a} \phi_{n-1}((n-1) a, t) .
$$

This constraint, together with the continuity condition,

$$
\phi_{n}(n a, t)=\phi_{n-1}(n a, t),
$$


are enough to specify the band structure. After some algebra, we find the consistency of (6.20), (6.22), and (6.23), demands

$$
\cos (p a)=\cos (k a)+\frac{4 \pi N \Delta^{2} k}{k^{2}-\omega_{0}^{2}} \sin (k a) .
$$

Here $p$ is to be understood as the photon momentum and $\omega=|k|$ as the photon energy.

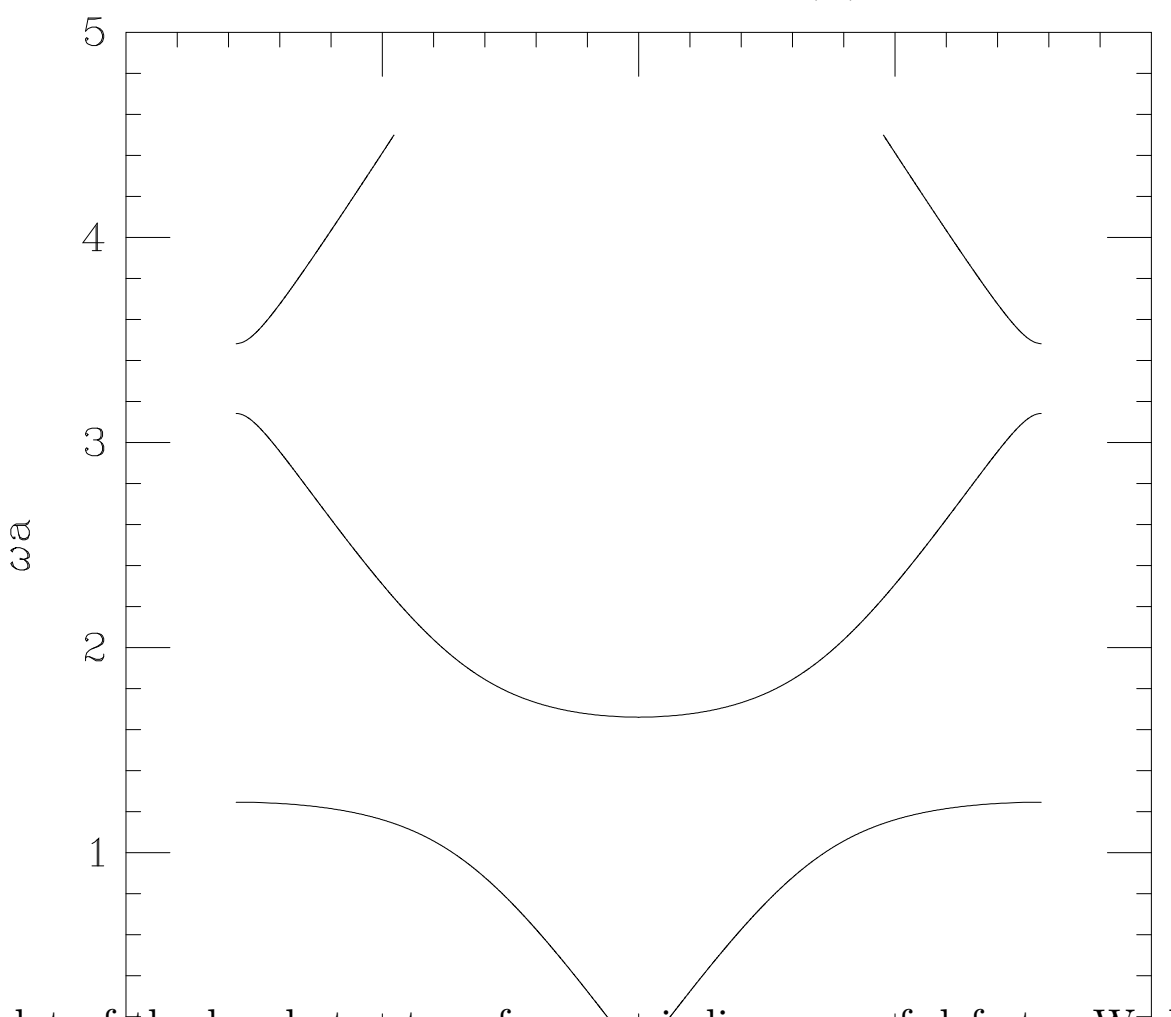

Figure 1: A plot of the band structure for a periodic array of defects. We have taken $\omega_{0}^{2} a^{2}=2$ and $44 \pi \omega_{0}^{2} \Delta^{2} a_{-2}^{3}=1$. We haye exagerated ${ }_{2}$ the value of 4 (or alternatively, underestimated $\omega_{0} a$ ) in order to maake the gaps visible.

A plot illustrating the band structure is given in Figure 1. In this plot are drawn the first two bands and a portion of the third in the reduced zone scheme. There are three notable features to the band structure: 1 ) in the lowest band, the energy, $k$, approaches 0 as $k \rightarrow 0 ; 2)$ there is a gap between the first and the second gap, a result of the resonance at $k=\omega_{0}$; and 3) there is a smaller gap between the second and third bands, more typical as it appears as a small perturbation about the edge of the first Brillouin zone.

The existence of a mode at $\omega=k=0$ results from gauge invariance. Because of the simplifications introduced by $\omega_{0} \Delta^{2}=\lambda^{2}$, the numerator in (6.26) vanishes as $k \rightarrow 0$. If the $\mathbf{A}^{2}$ term had been omitted from the action, we would have found that long wavelength, 
low energy photons cannot propagate, as if the photons had an imaginary mass. One, however, can ignore the $\mathbf{A}^{2}$ term if, at the same time, one makes the dipole approximation and couples the oscillators to the $\mathbf{E}$-field in place of the vector potential, A. This restores gauge invariance and so leads to the correct physics.

The effect of the pole at $k=\omega_{0}$ in (6.26) is to divide what would be the first unperturbed band $k=|k|,-\pi / a \leq k \leq \pi / a$ into two bands. The gap between these two bands, if small, is given by

$$
\Delta E=\left|\frac{4 \pi N \Delta^{2}}{\sin \left(\omega_{0} a\right)}\right| .
$$

The division into two branches is similar to what one sees in the dispersion relation for photons in an covalent crystal (see [12] - we will derive this dispersion relation in the next section). However there are two notable differences in [12]'s treatment from ours. There, the defects are coupled to the E-field and not to the vector potential (the dipole approximation) and the $\mathbf{A}^{2}$ term is ignored. The second difference is that the pole in [12] is shifted away from $k=\omega_{0}$. It seems plausible that this is a result of not treating all the oscillators in unison. When we treat an isolated defect in Section 3, we see a similar shift in the pole away from $\omega_{0}$ in $T(k)$ and $R(k)$.

The last feature of the band structure that bears comment is the gap between the second and third bands. This gap, far smaller than the previous one, is equal to

$$
\Delta E=\left|\frac{8 \pi^{2} \Delta^{2} N}{\pi^{2}-\omega_{0}^{2} a^{2}}\right| .
$$

We have ignored higher order terms in $\Delta^{2}$. Similar, but smaller, gaps will appear between the higher order bands. Again to lowest order in $\Delta^{2}$, the gap opening up at $\omega_{0} a=n \pi$ will equal (provided $\omega_{0} \neq n \pi / a$ )

$$
\Delta_{n} E=\left|\frac{8 N \pi^{2} \Delta^{2} n}{n^{2} \pi^{2}-\omega_{0}^{2} a^{2}}\right| .
$$

Such gaps are what one typically expects to find in the case of small perturbations: the band structure is only altered around the edges of the Brillouin zones. However there is a difference from the standard case: the opening up of the gaps is asymmetric about the energy $w=n \pi / a$ : the two solutions to (6.26) defining the gap are not $w=n \pi / a \pm \Delta_{n} E / 2$ (symmetric splitting) but $w=n \pi / a$ and $w=n \pi / a+\operatorname{sgn}\left(n^{2} \pi^{2}-\omega_{0}^{2} a^{2}\right) \Delta_{n} E$. 


\section{Continuum of Defects}

In this section we develop a theory describing a continuum set of defects living along the entire length of the fiber. As our starting point, we take a set of point defects distributed uniformly with linear density $\rho$ along the fiber. The corresponding action is then

$$
\begin{array}{rl}
S=\int d & t d x\left[\frac{1}{8 \pi}\left(\left(\partial_{t} \phi\right)^{2}-\left(\partial_{x} \phi\right)^{2}\right)\right. \\
& \left.+\sum_{k} \delta\left(x-x_{k}\right)\left(-i S_{k}^{-} \partial_{t} S_{k}^{+}-\omega_{0} S_{k}^{+} S_{k}^{-}+\lambda \sqrt{N}\left(S_{k}^{-}+S_{k}^{+}\right) \phi-N \Delta^{2} \phi^{2}\left(x_{k}\right)\right)\right] .
\end{array}
$$

We suppose the reduction to two dimensions, as in (6.17)-(6.20), has already been made. To take the continuum limit of this action, we introduce the field variables $S^{ \pm}(x)$ :

$$
S^{ \pm}(x)=\frac{1}{\sqrt{\rho}} \sum_{k} S_{k}^{ \pm} \delta\left(x-x_{k}\right)
$$

These then obey the expected commutation relations

$$
\left[S^{-}(x), S^{+}\left(x^{\prime}\right)\right]=\delta\left(x-x^{\prime}\right)
$$

and the action may be rewritten as

$$
\begin{gathered}
S=\int d t d x\left[\frac{1}{8 \pi}\left(\left(\partial_{t} \phi\right)^{2}-\left(\partial_{x} \phi\right)^{2}\right)-i S^{-}(x) \partial_{t} S^{+}(x)-\omega_{0} S^{+}(x) S^{-}(x)\right. \\
\left.+\sqrt{N \rho} \lambda\left(S^{-}(x)+S^{+}(x)\right) \phi-N \rho \Delta^{2} \phi^{2}(x)\right] .
\end{gathered}
$$

In doing so, we have smoothed the defect distribution by using the equality $\sum_{k} \delta\left(x-x_{k}\right)=$ $\rho$, a trick of Dirac's. As $\rho$ is typically $\sim 10^{6} \mathrm{~cm}^{-1}$, and $N \sim 10^{12}$, we have $N \rho \Delta^{2} / \omega_{0}^{2} \sim 10^{-4}$. This, again, is large enough to produce observable consequences.

From this action we are easily able to derive the exact dispersion relation. As in previous sections, we can write down an equation of motion involving $\phi$ alone:

$$
0=2 \omega_{0}^{2} \Delta^{2} N \rho \int_{-\infty}^{\infty} d \omega e^{i \omega t} \frac{\widetilde{\phi}(\omega, x)}{\omega^{2}-\omega_{0}^{2}}+2 N \rho \Delta^{2} \phi(x)+\frac{1}{4 \pi}\left(\partial_{t}^{2} \phi-\partial_{x}^{2} \phi\right) .
$$

The mode expansions of $\phi$ take the form

$$
\phi(x)=\int_{-\infty}^{\infty} d k \frac{1}{\sqrt{\omega(k)}}\left(A(k) e^{-i \omega(k) t+i k x}+A^{\dagger}(k) e^{i \omega(k) t-i k x}\right)
$$


Substituting this into the equation of motion gives us the dispersion relation

$$
\omega^{2}(k)=8 \pi N \rho \Delta^{2}+k^{2}+\frac{8 \pi N \rho \Delta^{2} \omega_{0}^{2}}{\omega^{2}(k)-\omega_{0}^{2}} .
$$

Like the band structure before, this has two branches. They are described by

$$
\omega_{ \pm}^{2}(k)=\frac{1}{2}\left[\left(\omega_{0}^{2}+8 \pi N \rho \Delta^{2}+k^{2}\right) \pm\left(\left(k^{2}+8 \pi N \rho \Delta^{2}-\omega_{0}^{2}\right)^{2}+32 \pi N \rho \Delta^{2} \omega_{0}^{2}\right)^{1 / 2}\right] .
$$

A plot of these two branches is found in the figure below:

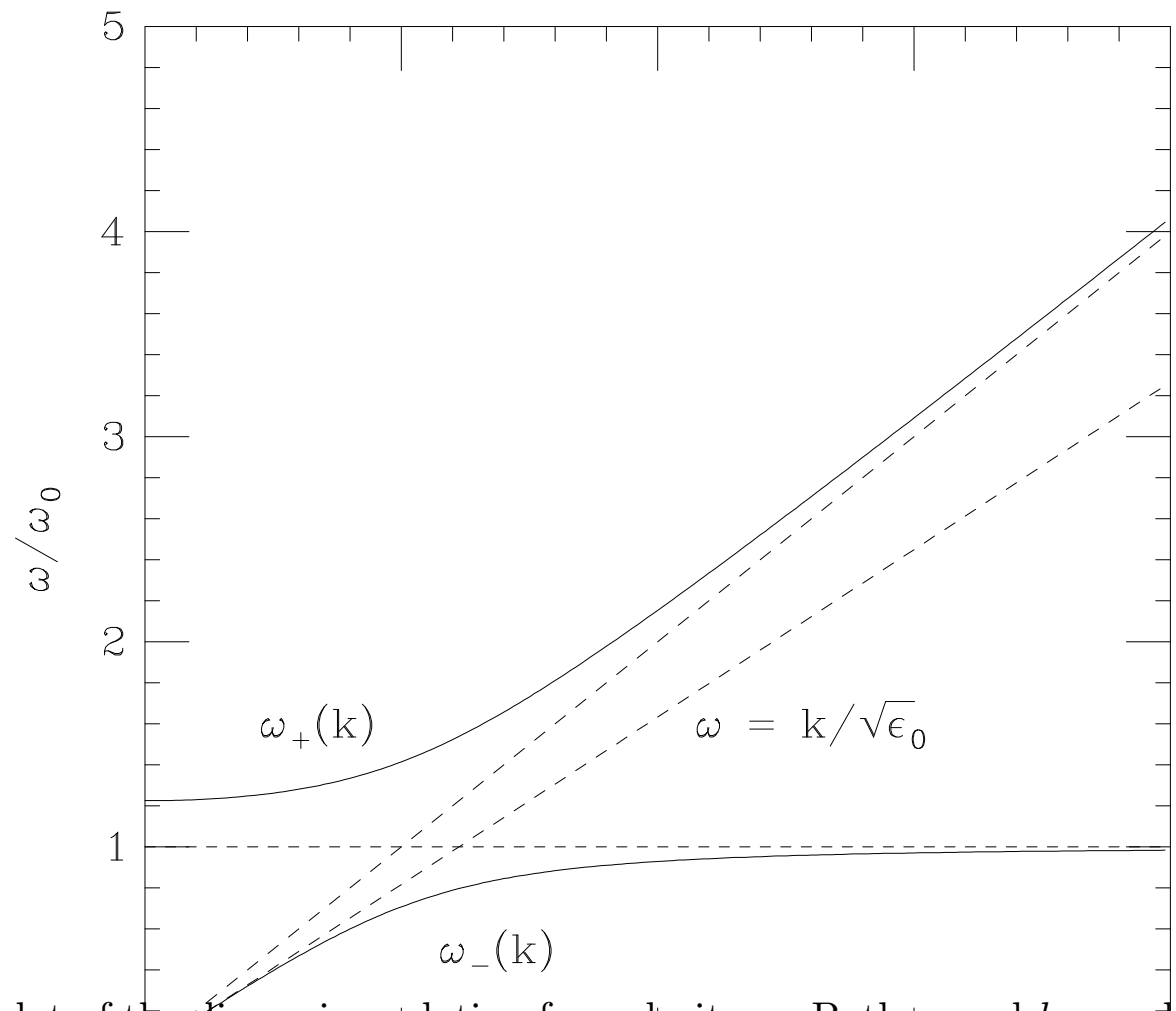

Figure 2: A plot of the dispersion relation for polaritons. Both $w$ and $k$ are $\mathrm{p}_{1}$ lotted in units of $\omega_{0}$. For this plot, we have taken $16 \pi N_{2} \rho \Delta^{2} \omega_{0}^{-2}=1.3$

$$
\mathrm{k} / \omega_{0}
$$

The modes described by these two branches of the dispersion relation are often referred to as polaritons.

As $k \rightarrow \infty$ both branches obtain asymptotic forms, as indicated by the dashed lines. The upper branch becomes photonic, $\omega=|k|$, as expected. At high energies the photons should pass undisturbed through a medium governed by far lower energy scales. The lower branch, on the other hand, approaches a constant, $\omega=\omega_{0}$, in this limit, becoming like an optical phonon. As $k \rightarrow 0$, the upper branch approaches a constant,

$$
\omega_{+}(0)=\sqrt{\omega_{0}^{2}+8 \pi N \rho \Delta^{2}} .
$$


A small gap thus separates these two branches. It is given by $\Delta E=\sqrt{\omega_{0}^{2}+8 \pi N \rho \Delta^{2}}-$ $\omega_{0}$. The lower branch, in contrast, becomes linear as $k \rightarrow 0$ and so describes photons propagating in a medium of dielectric constant

$$
\epsilon(0)=1+\frac{8 \pi N \rho \Delta^{2}}{\omega_{0}^{2}}
$$

Thus if we know $\epsilon(0)$, say experimentally, we can compute the effective coupling strength $N \rho \Delta^{2}$.

We are also able to compute the frequency dependent dielectric constant that gives rise to these dispersion relations. From (77.8) and $\epsilon(\omega) \omega^{2}=k^{2}$ we have

$$
\epsilon(\omega)=1+\frac{8 \pi N \rho \Delta^{2}}{\omega_{0}^{2}-\omega^{2}}
$$

The dielectric constant, $\epsilon(\omega)$ approaches $\pm \infty$ as $\omega \rightarrow \omega_{0}$. This asymptotic behaviour results in the two separate branches of polaritons. As $\omega \rightarrow \infty, \epsilon(\omega)$ approaches 1: as discussed previously, at energies far greater than $\omega_{0}$ the photons cease to see the oscillators.

\section{Impurities in the Presence of a Continuous Medium}

In previous sections, we have treated the defects as if embedded in the vacuum. The medium in between the defects has been assumed to have no dielectric properties, i.e. its dielectric constant, $\epsilon(\omega)$, has been set equal to 1 . This is not necessarily a bad approximation. The material out of which a fiber is constructed typically has a dielectric constant with little frequency dependence, i.e. $\epsilon(\omega)=\epsilon_{0}$, for a wide range of frequencies, $\hbar \omega_{0} \leq 10 \mathrm{eV}$. So by a judicious insertion of $\epsilon_{0}$ in the equations from previous sections, we can take into account the medium of propagation. However if we wish we can do better. As we have developed here a theory of a continuous medium, it is possible to solve the problem of a defect embedded in a medium with a frequency dependent dielectric constant. We will consider the defect to arise from atomic polarization. Alternatively we could consider the defect to be ionic in nature and suppose the natural frequency of the medium to be far below the energy scale where the ionic defect will also experience atomic polarization. 
The action describing such a defect is a sum of medium's action and the defect's action:

$$
\begin{aligned}
S=S_{\text {med }} & +S_{\mathrm{d}} \\
=\int d t d x & {\left[\frac{1}{8 \pi}\left(\left(\partial_{t} \phi\right)^{2}-\left(\partial_{x} \phi\right)^{2}\right)-i S^{-}(x) \partial_{t} S^{+}(x)-\omega_{m} S^{+}(x) S^{-}(x)\right.} \\
& \left.+\sqrt{N_{m} \rho_{m}} \lambda_{m}\left(S^{-}(x)+S^{+}(x)\right) \phi-N_{m} \rho_{m} \Delta_{m}^{2} \phi^{2}(x)\right] \\
& -\int d t\left[i S^{-} \partial_{t} S^{+}+\omega_{d} S^{+} S^{-}-\lambda_{d}\left(S^{-}+S^{+}\right) \phi+\Delta_{d}^{2} \phi^{2}\right] .
\end{aligned}
$$

We have labeled the defect and medium couplings with $d$ and $m$ subscripts respectively. The only difference in solving this theory from previous ones is in the nature of the mode expansions. Here the mode expansions are done in terms of polaritons:

$$
\phi(x)=\sum_{j=+,-} \int_{-\infty}^{\infty} d k \frac{1}{\sqrt{w_{j}(k)}}\left(A_{j}(k) e^{-i \omega_{j}(k) t+i k x}+A_{j}^{\dagger}(k) e^{i \omega_{j}(k)-i k x}\right) .
$$

The sum reflects that the dispersion relation has two branches. So the scattering matrices for the polaritons off the defect are similar to those of (3.12) with $\omega(k)=|k|$ replaced by $\omega(k)=\omega_{ \pm}(k)$ (where $\omega_{ \pm}$are given from $(7.8)$ by replacing $\omega_{0}$ with $\omega_{m}, N \rho$ with $N_{m} \rho_{m}$, and $\Delta$ with $\Delta_{m}$ ). Thus we have

$$
\begin{aligned}
T_{ \pm}(k) & =\frac{i k\left(\omega_{ \pm}^{2}(k)-\omega_{d}^{2}\right)}{i k\left(\omega_{ \pm}^{2}(k)-\omega_{d}^{2}\right)-4 \pi \Delta_{d}^{2} \omega_{ \pm}^{2}(k)} \\
R_{ \pm}(k) & =\frac{4 \pi \Delta_{d}^{2} \omega_{ \pm}^{2}(k)}{i k\left(\omega_{ \pm}^{2}(k)-\omega_{d}^{2}\right)-4 \pi \Delta_{d}^{2} \omega_{ \pm}^{2}(k)}
\end{aligned}
$$

We now need to index $T$ and $R$ to indicate which branch the polariton is on.

Similarly we can imagine an array of defects imbedded in the continuous medium. The band structure is then derived from $(6.26)$ by replacing the free dispersion relation with $\omega_{ \pm}$:

$$
\cos \left(p_{ \pm} a\right)=\cos (k a)+\frac{4 \pi N_{d} \Delta_{d}^{2} \omega_{ \pm}^{2}(k)}{k\left(\omega_{ \pm}^{2}(k)-\omega_{d}^{2}\right)} \sin (k a),
$$

where $N_{d}$ is the number of defects in a section transverse to the fiber. Here $p_{ \pm}$is the crystal momentum for polaritons on the upper/lower branch of the dispersion relation. For a given $p_{ \pm}$, a set of $k^{\prime}$ s, $\left\{k_{i}\right\}$, satisfy this equation. The energies corresponding to this $p_{ \pm}$are then $\left\{\omega_{ \pm}\left(k_{i}\right)\right\}$. A plot of the band structure is given in Figure 3 below. 


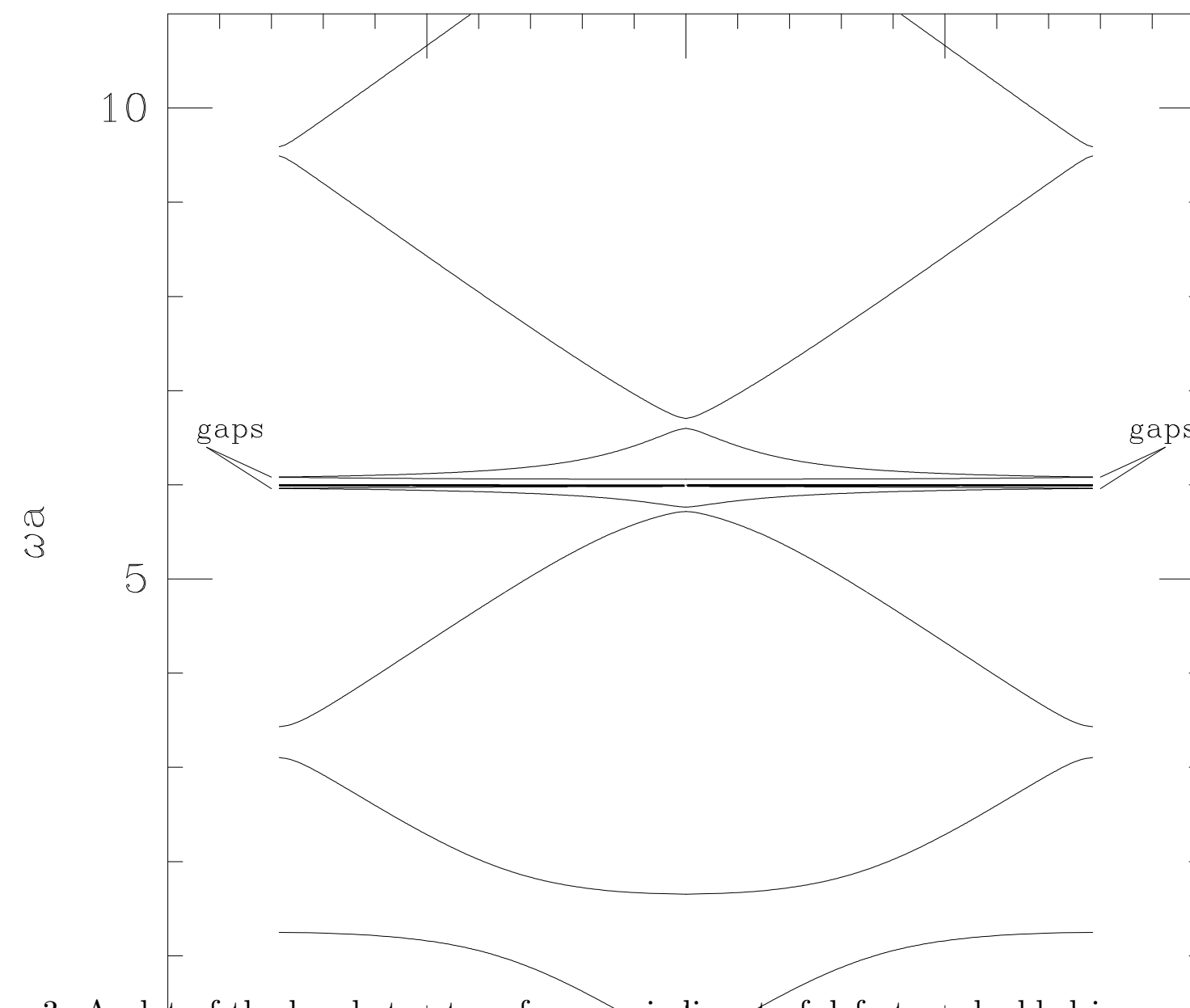

Figure 3: A plot of the band structure for a periodic set of defects embedded in a material governed by the dispersion relation in (7.7). For the defects we have again taken $\omega_{d}^{2} a^{2}=\overline{2}, \mathbb{1}_{4 \pi} N_{d} \omega_{d}^{2} \Delta^{2} a^{3} \equiv 2$. For the parahneters of the me⿻i⿵um, we have 4 taken $\omega_{m} a=6$ and $32 \pi N_{m} \rho_{m} \Delta^{2} \omega_{m}^{2} a^{4}=100$. TRe gaps that are too small to be seen have been explicitly marked out.

The gap between $\omega_{+}(k)$ and $\omega_{-}(k)$ forces the bands corresponding to these two branches to be separated by this same gap. The bands of $\omega_{-}(k)$ are found below $\omega_{m}$ while those of $\omega_{+}(k)$ are bounded below by $\sqrt{\omega_{m}^{2}+8 \pi N_{m} \rho_{m} \Delta^{2}}$. The lower set of bands away from $\omega_{m}$ are similar to those found in Section 6. For small $k$ and $\omega$, the spectrum is linear; there is a large gap between the first and second bands; and there are smaller gaps between the higher order bands. The similarity results from $\omega_{-}(k)$ being photonic for small $k$. Close to $\omega_{m}$ the bands of $\omega_{-}(k)$ accumulate. They are of an infinite number, each separated by vanishingly small energies. The existence of such bands owes itself to the asymptotic form of $\omega_{-}(k)$ : as $k \rightarrow \infty, \omega_{-}$approaches a constant. The upper set of bands 
are photonic away from $\omega_{m}$ (provided $\omega_{m}>\omega_{d}$ ) as $w_{+}(k)$ is photonic for large energies. Only near $\omega_{m}$ do the bands of $\omega_{+}(k)$ deviate from linearity, a result of $\omega_{+}(0) \neq 0$.

The gap between the first and second band of $\omega_{-}(k)$ is described as in (6.27):

$$
\Delta E=\left|\frac{4 \pi N_{d} \Delta_{d}^{2}}{\sqrt{\epsilon\left(\omega_{d}\right)} \sin \left(\sqrt{\epsilon\left(\omega_{d}\right)} \omega_{d} a\right)}\right|,
$$

where $\epsilon\left(\omega_{d}\right)$ is given by (7.11). The gaps opening up at $p=n \pi$ for both sets of bands are given by

$$
\begin{gathered}
\Delta_{n} E_{ \pm}=\left|\gamma_{ \pm}(n)\right| \partial_{k} \omega_{ \pm}(n \pi / a) \\
\gamma_{ \pm}=\frac{8 N_{d} \Delta_{d}^{2} \omega_{ \pm}^{2}(n \pi / a)}{n\left(\omega_{ \pm}^{2}(n \pi / a)-\omega_{d}^{2}\right)} ; \\
\omega_{+}(k)=k+O\left(\Delta_{m}^{2}\right) ; \quad \omega_{-}(k)=\omega_{m}+O\left(\Delta_{m}^{2}\right) ; \\
\partial_{k} \omega_{+}(k)=1+O\left(\Delta_{m}^{2}\right) ; \quad \partial_{k} \omega_{-}(k)=\frac{8 \pi \Delta_{m}^{2} N_{m} \rho_{m} \omega_{m} k}{\left(k^{2}-\omega_{m}^{2}\right)^{2}} .
\end{gathered}
$$

We have again only kept terms to lowest order in $O\left(\Delta_{d}^{2}\right)$ and $O\left(\Delta_{m}^{2}\right)$. These gaps, as with their counterparts in Section 6 , are not symmetric about $\omega_{ \pm}(n \pi)$. Rather the gap is defined by $\omega=\omega_{ \pm}(n \pi / a)$ and $\omega=\omega_{ \pm}(n \pi / a)+\operatorname{sgn}\left(\omega_{ \pm}(n \pi / a)^{2}-\omega_{d}^{2}\right) \Delta_{n} E_{ \pm}$. 


\section{References}

[1] G. Delfino, G. Mussardo, and P. Simonetti, Nucl. Phys. B432 (1994) 518.

[2] S. Ghoshal and A. Zamolodchikov, Int. J. Mod. Phys. A9 (1994) 3841.

[3] A. LeClair, G. Mussardo, H. Saleur, S. Skorik, Nucl. Phys. B453 (1995) 581.

[4] V. I. Rupasov, JETP Lett. 36 (1982) 142.

[5] A. LeClair, F. Lesage, S. Lukyanov, H. Saleur, Exact Solution of the Maxwell-Bloch Theory in Quantum Optics, CLNS preprint.

[6] A. LeClair, QED for a Fibrillar Medium of Two-Level Atoms, hep-th 9604100.

[7] D.F. Walls, G.J. Milburn, Quantum Optics, Springer-Verlag, New York, 1994, ch. $6.1)$.

[8] P. Fendley, A. W. W. Ludwig, H. Saleur., Phys. Rev. Lett 74 (1995) 3005.

[9] R. J. Glauber, Phys. Rev. 130 (1963) 2529.

[10] G. Rickayzen, Green's Functions and Condensed Matter, Academic Press, NY, 1980 .

[11] L. Allen and J. H. Eberly, Optical Resonance and Two-Level Atoms, Dover, New York, 1987.

[12] N. Ashcroft and D. Mermin, Solid State Physics, W. B. Saunder Company, Philadelphia, 1976, ch. 27. 